\title{
Active Site Histidines Link Conformational Dynamics with Catalysis on Anti-Infective Target 1-Deoxy-o-xylulose 5- Phosphate Synthase
}

\author{
Alicia A. DeColli ${ }^{\dagger}, \mathrm{Xu}_{\text {Zhang }}^{\ddagger}$, Kathryn L. Heflin ${ }^{\dagger} \S$, Frank Jordan ${ }^{\ddagger}$, Caren L. Freel Meyers ${ }^{*}, \dagger$ \\ tDepartment of Pharmacology and Molecular Sciences, The Johns Hopkins University School of \\ Medicine, Baltimore, Maryland 21205, United States \\ ¥Department of Chemistry, Rutgers University, Newark, New Jersey 07102, United States
}

\begin{abstract}
The product of 1-deoxy-D-xyluose 5-phosphate (DXP) synthase, DXP, feeds into the bacterial biosynthesis of isoprenoids, thiamin diphosphate (ThDP), and pyridoxal phosphate. DXP is essential for human pathogens but not utilized by humans; thus, DXP synthase is an attractive antiinfective target. The unique ThDP-dependent mechanism and structure of DXP synthase offer ideal opportunities for selective targeting. Upon reaction with pyruvate, DXP synthase uniquely stabilizes the predecarboxylation intermediate, $\mathrm{C} 2 a$-lactylThDP (LThDP), in a closed conformation. Subsequent binding of D-glyceraldehyde 3-phosphate induces an open conformation that is proposed to destabilize LThDP, triggering decarboxylation. Evidence for the closed and open conformations has been revealed by hydrogen-deuterium exchange mass spectrometry and X-ray crystallography, which indicate that $\mathrm{H} 49$ and $\mathrm{H} 299$ are involved in conformational dynamics and movement of the fork and spoon motifs away from the active site is important for the closedto-open transition. Interestingly, H49 and H299 are critical for DXP formation and interact with the predecarboxylation intermediate in the closed conformation. H299 is removed from the active
\end{abstract}

\footnotetext{
*Corresponding Author Telephone: 410-502-4807. Fax: 410-955-3023. cmeyers@jhmi.edu. Author Contributions

C.L.F.M. and A.A.D. conceived and designed the study and prepared the manuscript. K.L.H. performed site-directed mutagenesis and performed preliminary biochemical characterization of all variants under aerobic conditions. X.Z. designed, conducted, and analyzed HDX-MS experiments. A.A.D. designed, conducted, and interpreted all of the other experiments. K.L.H., X.Z., and F.J. contributed to the preparation of the manuscript. All authors reviewed the results and approved the final version of the manuscript.

\$Present Address

iCubate, Huntsville, AL 35806.

Supporting Information

The Supporting Information is available free of charge at https://pubs.acs.org/doi/10.1021/acs.biochem.9b00878.

General methods, oxygenase activity of H49 and H299 variants, determination of the secondary structure of DXP synthase enzymes, determination of the apparent $T_{\mathrm{m}}$ of DXP synthase enzymes, oxygenase activity of $\mathrm{H} 49$ and $\mathrm{H} 299$ variants (Figure S1), representative Michaelis-Menten curves for the wild type and variants under aerobic conditions (Figure S2), representative Michaelis-Menten curves for the wild type and variants under anaerobic conditions (Figure S3), representative Morrison and IC50 plots for MAP inhibition against the wild type and variants (Figure $\mathrm{S} 4$ ), comparison of the secondary structure and apparent $T_{\mathrm{m}}$ of DXP synthase enzymes (Figure S5), $\mathrm{m} / \mathrm{z}$ plots of HDX of peptide 183-199 on the wild type, H49A, and H299N (Figure S6), $\mathrm{m} / \mathrm{z}$ plots of HDX of peptide 188-199 on the wild type, H49A, and H299N (Figure S7), m/z plots of HDX of peptide 278-298 on the wild type, H49A, and H299A (Figure S8), and aerobic characterization of the wild type, H49, and H299 DXP synthase (Table S1) (PDF)

Accession Codes

E. coli DXP synthase, Uniprot P77488, NCBI Q0TKM1; D. radiodurans DXP synthase, Uniprot Q9RUB5, NCBI Q9RUB5; E. coli

E1-PDH, Uniprot P0AFG8, NCBI PJI63074; E. coli MEP synthase, Uniprot P45568, NCBI ATZ31749.

The authors declare no competing financial interest.
} 
site in the open conformation of the postdecarboxylation state. In this study, we show that substitution at $\mathrm{H} 49$ and $\mathrm{H} 299$ negatively impacts LThDP formation by shifting the conformational equilibrium of DXP synthase toward an open conformation. We also present a method for monitoring the dynamics of the spoon motif that uncovered a previously undetected role for H49 in coordinating the closed conformation. Overall, our results suggest that $\mathrm{H} 49$ and $\mathrm{H} 299$ are critical for the closed, predecarboxylation state providing the first direct link between catalysis and conformational dynamics.

\section{Graphical Abstract}

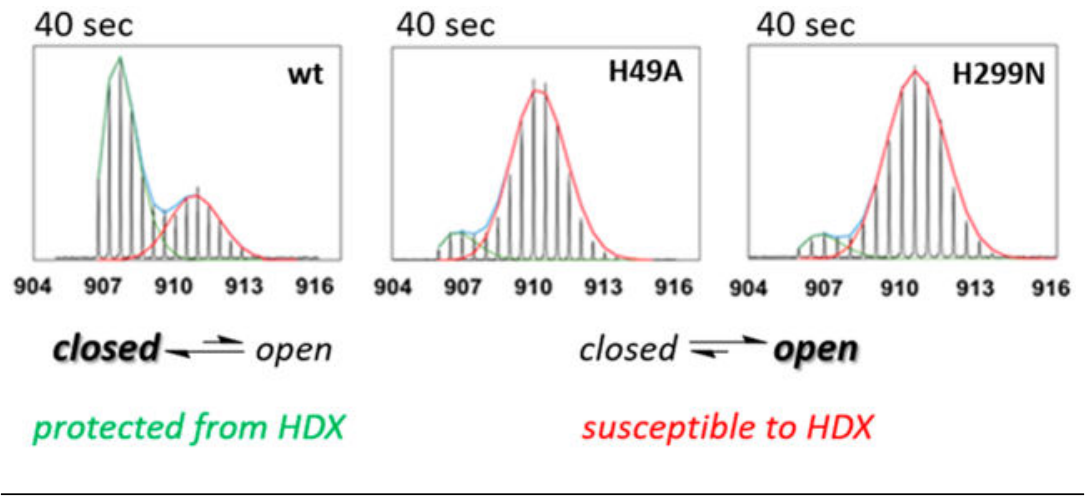

1-Deoxy-D-xylulose 5-phosphate (DXP) synthase catalyzes the thiamin diphosphate (ThDP)-dependent decarboxylation of pyruvate and subsequent carboligation to Dglyceraldehyde 3-phosphate (D-GAP) (Figure 1). DXP is an essential intermediate in bacterial metabolism feeding into the biosynthesis of ThDP, pyridoxal phosphate (PLP), and isoprenoids (Figure 1). ${ }^{1-4}$ Because DXP is an indispensable bacterial metabolite not utilized by humans, its inhibition should be selectively toxic to human pathogens. To this end, studies of DXP synthase have discovered unique structural and mechanistic features that set it apart from others in the ThDP enzyme family, enabling the design of potent, selective inhibitors. ${ }^{5-9}$ Early structural studies revealed a unique domain arrangement and novel positioning of large active sites on DXP synthase, distinct from transketolase (TK) and the E1 component of the Escherichia coli pyruvate dehydrogenase complex (E1-PDH). ${ }^{10,11} \mathrm{In}$ addition, mechanistic studies have demonstrated a unique mechanism for DXP synthase in which the first enzyme-bound intermediate, C2a-lactylThDP [LThDP (Figure 1)], is stabilized in the absence of an acceptor substrate. ${ }^{12}$ Ternary complex formation upon binding of D-GAP to the E-LThDP complex is required to facilitate LThDP decarboxylation (Figure 1, steps 3 and 4). ${ }^{12-14}$ Characterization of DXP synthase oxygenase activity revealed a similar mechanism of LThDP decarboxylation induced by $\mathrm{O}_{2}$. Thus, D-GAP and $\mathrm{O}_{2}$ are both triggers of LThDP decarboxylation on DXP synthase. This preferred order random sequential mechanism is unique among ThDP-dependent pyruvate decarboxylase enzymes, as most others in this family are known to catalyze ping-pong mechanisms in which LThDP decarboxylation readily occurs in the absence of an acceptor substrate. ${ }^{13-19}$ Accordingly, selective inhibition strategies developed by our group thus far have targeted the large active site of the unique E-LThDP conformation of DXP synthase. 
Ternary complex formation involved in D-GAP-induced decarboxylation of LThDP [ELThDP-GAP (Figure 1, step 4)] represents another targetable feature of DXP synthase. Although this mechanism is not fully understood, we and others have demonstrated substrate-dependent conformational changes that are thought to underlie LThDP stabilization on DXP synthase and to drive LThDP decarboxylation. ${ }^{18,20}$ Hydrogendeuterium exchange mass spectrometry (HDX-MS) in the presence and absence of a substrate analogue or product revealed conformational flexibility in three regions near the active site (residues 42-58, 183-199, and 278-298), here termed "EX1 regions" as they are characterized by an EX1 mechanism of $\mathrm{H}-\mathrm{D}$ exchange resulting in the simultaneous appearance of both a closed and an open conformation. ${ }^{18}$ In the absence of substrates, these regions exist in an equilibrium between open and closed forms (Figure 1). ${ }^{18}$ In the presence of methylacetylphosphonate (MAP), a pyruvate analogue that reacts with ThDP to form a stable LThDP mimic, C2 $a$-phosphonolactylThDP (PLThDP), the equilibrium shifts to favor a closed form that is protected from $\mathrm{H}-\mathrm{D}$ exchange. ${ }^{18}$ The closed conformation is presumed to stabilize LThDP in the absence of D-GAP. In contrast, binding of D-GAP or DXP induces an open conformation that is susceptible to H-D exchange in these regions. ${ }^{18}$ These results suggest a role for the flexible EX1 regions in catalysis and hint at residues that may link conformational dynamics to the unique catalytic mechanism of DXP synthase. Two active site histidine residues (H49 and $\mathrm{H} 299$ on E. coli DXP synthase) proposed to be important for pyruvate binding and catalysis ${ }^{19,21,22}$ reside within (H49) and adjacent to (H299) EX1 regions 1 and 3, respectively, suggesting they may play additional roles in coordinating the pyruvate-dependent conformational change to a closed form.

We have recently reported the first ligand-bound crystal structures of Deinococcus radiodurans DXP synthase that provide further support for this hypothesis. ${ }^{16}$ Consistent with mutagenesis results, ${ }^{19,21,22}$ the structure of DXP synthase with PLThDP bound shows that H51 and H304 (analogous to E. coli DXP synthase residues H49 and H299, respectively) are within hydrogen bonding distance of the phosphonyl group of PLThDP in the closed conformation (Figure 2A,C). By extension, H51 and H304 are predicted to interact with and stabilize the carboxyl group of the predecarboxylation intermediate LThDP. The EX1 regions identified by HDX-MS are blocked in the closed conformation by two structural motifs, termed the fork (residues 292-306, teal) and spoon (residues 307-319, orange) motifs, restricting solvent accessibility (Figure 2). The structure of DXP synthase bound to a postdecarboxylation intermediate was also determined (tentatively assigned as the enamine or its protonated form with the caveat that the protonation and oxidation state could not be unequivocally determined at the reported resolution). In contrast to the predecarboxylation form, this structure exists in an open conformation in which the fork motif is disordered, and the spoon motif is repositioned to one side of the active site cleft. As illustrated in panels B and D of Figure 2, this structural change effectively removes H304 from the active site and dramatically increases solvent accessibility (and thus the level of H-D exchange) in the EX1 regions identified by HDX-MS. These static snapshots of closed and open conformations suggest that removal of $\mathrm{H} 304$ from the active site in the postdecarboxylation state coincides with LThDP decarboxylation.

Taken together, the HDX-MS and X-ray crystallography results are consistent with a role of $D I H 304(E c H 299)$ in coordinating the conformational change to the closed form that is 
required for LThDP formation and stabilization and support the involvement of H304 in conformational dynamics required for DXP synthase to undergo the transition from the closed (predecarboxylation) to open (postdecarboxylation) state. We have hypothesized a similar role for EcH49 ( $D r H 51)$ based on its proximity to EX1 regions identified by HDXMS; however, the pre- and postdecarboxylation crystal structures do not reveal dramatic movements of $\mathrm{H} 51$ between these two states.

The study reported herein has sought to establish a role for these histidine residues in DXP synthase conformation and a link between binding/catalysis and conformational changes that occur on DXP synthase. We have employed a combination of mutagenesis and structural methods [HDX-MS, limited proteolysis, and circular dichroism (CD)] to correlate the effects of substituting H49 and H299 on E. coli DXP synthase conformational dynamics and catalysis. The kinetic parameters of these variants were determined under anaerobic conditions to exclude confounding effects from the oxidative decarboxylation of pyruvate ${ }^{13}$ and were found to be comparable to kinetic parameters determined under aerobic conditions; substitution of either residue dramatically reduces the catalytic efficiency, with substitutions at $\mathrm{H} 299$ having the greatest impact. Limited trypsinolysis was employed as a new tool together with HDX-MS analysis to demonstrate that substitution of either H49 or H299 significantly shifts the conformational equilibrium of DXP synthase, in a manner that negatively impacts catalysis. Specifically, we show that, in contrast to the wild type enzyme, H49 and H299 variants adopt an open conformation even in the presence of the PLThDP, a mimic of the predecarboxylation intermediate LThDP that is known to shift the equilibrium of wild type DXP synthase to favor the closed conformation for LThDP stabilization. These results are significant because they show that substitution of either $\mathrm{H} 49$ or $\mathrm{H} 299$ decreases catalytic efficiency by altering the conformational equilibrium, providing to the best of our knowledge the first direct connection between conformational changes and catalysis on DXP synthase. In addition, our results reveal a critical role of $\mathrm{H} 49$ in coordinating the closed conformation required for LThDP formation and stabilization, which is not obvious from the structures of open and closed conformations. Taken together, these results provide key insights into conformational dynamics on DXP synthase that will be important for the design of selective inhibition strategies.

\section{MATERIALS AND METHODS}

\section{General Methods.}

Unless otherwise noted, all reagents were obtained from commercial sources. All DNA primers were purchased from Integrated DNA Technologies. E. coli DXP synthase and $E$. coli MEP synthase (IspC) were overexpressed and purified as reported previously. ${ }^{23,40}$ Anaerobic spectropho-tometric analyses were performed on a Tecan infinite M nano UV/ visible plate reader (Switzerland) that is housed inside a Coy Laboratory Products (Grass Lake, MI) vinyl anaerobic chamber. Anaerobic conditions for experiments that were conducted outside of the chamber (e.g., CD) were established in the anaerobic chamber, and anaerobic solutions were transferred to airtight, septum-capped vials or cuvettes. For steady state CD studies, spectra were recorded on an Aviv (Lakewood, NJ) 420 CD spectrometer. Trypsin digest peptides were sequenced by the Taplin Mass Spectrometry Facility at Harvard 
University (Cambridge, MA). Intact masses of trypsin digest peptides were determined by the Mass Spectrometry Core at The Johns Hopkins University School of Medicine. HDXMS experiments were carried out on a 7T Bruker Daltonics Fourier Transform Mass Spectrometer.

\section{Construction of H49 and H299 DXP Synthase Variants.}

The construction of H49A and H299A DXP synthase variants has been reported previously.

${ }^{41}$ A similar approach, adapted from Agilent's Quikchange mutagenesis protocol, was initially used to generate other DXP synthase histidine variants. Polymerase chain reaction (PCR) mixtures (total volume of $50 \mu \mathrm{L}$ ) contained 1xbuffer (NEB), $200 \mu \mathrm{M}$ dNTPs (Agilent), $1 \mathrm{ng} / \mu \mathrm{L}$ template, $1 \mu \mathrm{M}$ primer, and $1 \mu \mathrm{L}$ ( 2 units) of Phusion polymerase (NEB). The $d x s$-pET37b plasmid (wild type DXP synthase) was used as the template and purified from $E$. coli Top 10 competent cells harboring the plasmid of interest using a Qiagen QIAprep Spin Miniprep kit. Primer sequences were designed generally using Agilent's Quikchange primer design tool and purchased from IDT (substitutions underlined): H49N, 5'-CGTTCCAGCGGGAACTTCGCCTCCG-3' and 5'CGGAGGCGAAGTTCCCGCTGGAACG-3'; H299N, 5' GAAAAAGACCCGATCACTTTCAACGC- $3^{\prime}$ and $5^{\prime}$ TTAGGCACGGCGTTGAAAGTGATCGG- $3^{\prime}$. The PCR was set up as follows: $98^{\circ} \mathrm{C}$ for $30 \mathrm{~s}$, followed by 25 cycles of $98^{\circ} \mathrm{C}$ for $10 \mathrm{~s}$, an annealing temperature of $52^{\circ} \mathrm{C}(\mathrm{H} 49 \mathrm{~N})$ or $56{ }^{\circ} \mathrm{C}(\mathrm{H} 299 \mathrm{~N})$ for $30 \mathrm{~s}$, and $72{ }^{\circ} \mathrm{C}$ for $10 \mathrm{~min}$, followed by a final $10 \mathrm{~min}$ elongation at $72{ }^{\circ} \mathrm{C}$. The PCR products were digested with DpnI (NEB) for 2-4 h. DpnI-digested products were transformed into E. coli Top 10 or XL1 Blue competent cells using a standard heat shock protocol. Constructs were fully sequenced by Genewiz to confirm the presence of the desired substitution and C-His 8 tag. Constructs were subsequently transformed into $E$. coli BL21 (DE3) competent cells for protein overexpression.

\section{Steady State Characterization of DXP Formation by DXP Synthase Enzymes.}

DXP formation was monitored using the IspC-coupled assay previously described ${ }^{5,23}$ with the following modifications. All solutions were prepared in the anaerobic chamber. When pyruvate was the varied substrate $(0.0075-1 \mathrm{mM}$ for wild type and $0.125-10 \mathrm{mM}$ for $\mathrm{H} 49$ and $\mathrm{H} 299$ variants), the D-GAP concentration was held constant at $500 \mu \mathrm{M}$. When D-GAP was the varied substrate $(0.0075-1 \mathrm{mM})$, the pyruvate concentration was held constant at $1 \mathrm{mM}$ for the wild type or $5 \mathrm{mM}$ for $\mathrm{H} 49$ and $\mathrm{H} 299$ variants (DXP synthase catalyzes confounding acetolactate formation at a pyruvate concentration of $>5 \mathrm{mM}) .{ }^{17} \mathrm{~A}$ final enzyme concentration of $150 \mathrm{nM}$ (wild type), $500 \mathrm{nM}$ (H49A, H49N, and H299N), or $2 \mu \mathrm{M}$ (H299A) was used. Reactions were conducted in a 96-well plate, and DXP formation was initiated upon the addition of $100 \mu \mathrm{L}$ of a $2 \times$ substrate solution to $100 \mu \mathrm{L}$ of a $2 \times$ enzyme solution containing DXP synthase (final concentrations described above), IspC (final concentration of $4 \mu \mathrm{M}$ ), and NADPH (final concentration of $300 \mu \mathrm{M}$ ) in DXP synthase reaction buffer [50 mM 4-(2-hydroxyethyl)-1-piperazineethanesulfonic acid (HEPES) (pH 8), $100 \mathrm{mM} \mathrm{NaCl}, 2 \mathrm{mM} \mathrm{MgCl}_{2}$, and $1 \mathrm{mM} \mathrm{ThDP]}$. The disappearance of NADPH was monitored at $340 \mathrm{~nm}$ at chamber temperature $\left(25-28{ }^{\circ} \mathrm{C}\right)$. Initial rates of NADPH depletion were used to estimate rates of DXP formation. Plots of the initial rate of DXP formation 
versus substrate concentration were fit to the Michaelis-Menten equation in GraphPad Prism version 6. All experiments were conducted in triplicate.

\section{Determination of $K_{i}$ and Mode of Inhibition of MAP against DXP Synthase Enzymes.}

All reactions were conducted in a 96-well plate in the anaerobic chamber at chamber temperature $\left(25-28{ }^{\circ} \mathrm{C}\right)$. Inhibition constants $\left(K_{\mathrm{i}}\right)$ and the mode of inhibition were determined as previously reported ${ }^{5}$ with the following deviations. For $K_{\mathrm{i}}$ determination, DGAP $(500 \mu \mathrm{M})$, pyruvate $\left(2 K_{\mathrm{m}}{ }^{\text {pymvate }}, 80 \mu \mathrm{M}\right.$ for wild type and $2 \mathrm{mM}$ for $\mathrm{H} 49$ and $\mathrm{H} 299$ variants), and MAP (varied from 0.1 to $75 \mu \mathrm{M}$ against the wild type and from 0.05 to $10 \mathrm{mM}$ against $\mathrm{H} 49$ and $\mathrm{H} 299$ variants) were simultaneously added to the enzyme solution described above. All enzyme concentrations were the same as described above with the exception of $\mathrm{H} 299 \mathrm{~A}(3 \mu \mathrm{M})$ and $\mathrm{H} 299 \mathrm{~N}(1.5 \mu \mathrm{M})$. Initial rates of DXP formation were determined from initial rates of NADPH depletion and plotted against MAP concentration. These plots were fit to the Morrison equation ${ }^{42}$ to determine inhibition constants. The Morrison equation is defined by eq 1 , where initial reaction rates $(v)$ are measured at varying inhibitor concentrations, $\left[\mathrm{I}_{\mathrm{T}}\right.$, and a constant enzyme concentration, $[\mathrm{E}]_{\mathrm{T}}$.

$$
v=1-\frac{[\mathrm{E}]_{\mathrm{T}}+[\mathrm{I}]_{\mathrm{T}}+K_{\mathrm{i}}^{\mathrm{app}}-\sqrt{\left([\mathrm{E}]_{\mathrm{T}}+[\mathrm{I}]_{\mathrm{T}}+K_{\mathrm{i}}^{\text {app }}\right)^{2}-4[\mathrm{E}]_{\mathrm{T}}[\mathrm{I}]_{\mathrm{T}}}}{2[\mathrm{E}]_{\mathrm{T}}}
$$

$K_{\mathrm{i}}^{\mathrm{app}}$ is described by eq 2, where $[\mathrm{S}]$ is the substrate concentration and $K_{\mathrm{m}}$ is the MichaelisMenten constant determined for that substrate.

$$
K_{\mathrm{i}}^{\mathrm{app}}=K_{\mathrm{i}}\left(1+\frac{[\mathrm{S}]}{K_{\mathrm{m}}}\right)
$$

Experiments to determine the mode of inhibition with respect to pyruvate were conducted like those for $K_{\mathrm{i}}$; however, the $\mathrm{IC}_{50}$ at $2 K_{\mathrm{m}}$ pyruvate (described above) and $10 K_{\mathrm{m}}$ pyruvate (400 $\mu \mathrm{M}$ for the wild type and $10 \mathrm{mM}$ for $\mathrm{H} 49$ and $\mathrm{H} 299$ variants) was plotted against [substrate] $/ K_{\mathrm{m}}{ }^{\text {pyruvate }}$. At $10 K_{\mathrm{m}}{ }^{\text {pyruvate }}$, the MAP concentration was varied from 0.5 to 250 $\mu \mathrm{M}$ against the wild type and from 0.1 to $45 \mathrm{mM}$ against $\mathrm{H} 49$ and $\mathrm{H} 299$ variants. All experiments were conducted in triplicate.

\section{Steady State CD Analysis of DXP Synthase Enzymes in the Absence and Presence of Pyruvate.}

All solutions were deoxygenated and prepared in the anaerobic chamber prior to removal from the chamber in airtight septum-capped vials or cuvettes for transfer via gastight syringe. Initially, enzyme-only spectra of wild type DXP synthase or variants $(30 \mu \mathrm{M})$ in 50 mM HEPES ( $\mathrm{pH} 8$ ), $100 \mathrm{mM} \mathrm{NaCl}, 1 \mathrm{mM} \mathrm{MgCl}_{2}$, and $0.2 \mathrm{mM}$ ThDP were obtained from 280 to $450 \mathrm{~nm}$ with a $1 \mathrm{~nm}$ step and $2 \mathrm{~s}$ averaging time at $4{ }^{\circ} \mathrm{C}$. The same scan method was repeated during a titration of pyruvate (7-400 $\mu \mathrm{M}$ for the wild type or $12.5-3147 \mu \mathrm{M}$ for variants) after each addition of pyruvate via syringe. All titrations were conducted in triplicate for each variant and wild type DXP synthase. Single titrations of H49A/H49N 
were conducted on the same day to confirm observed differences in CD magnitudes for conservative and nonconservative substitutions. Similarly, single titrations of H299A/H299N were conducted on the same day.

\section{HDX-MS Experiments and Data Analysis.}

Prior to H-D exchange, DXP synthase was exchanged into $50 \mathrm{mM}$ HEPES (pH 8.0) containing $50 \mathrm{mM} \mathrm{NaCl}, 0.2 \mathrm{mM} \mathrm{ThDP}$, and $1 \mathrm{mM} \mathrm{MgCl}_{2}$, and then the protein concentration was adjusted to $80 \mu \mathrm{M}$. The concentrated DXP synthase $(80 \mu \mathrm{M})$ was incubated for $10 \mathrm{~min}$ at $25^{\circ} \mathrm{C}$ prior to initiation of the HDX experiments. The HDX experiments were initiated by mixing $15 \mu \mathrm{L}$ of the protein samples with $285 \mu \mathrm{L}$ of $\mathrm{D}_{2} \mathrm{O}$ buffer to a final concentration of $95 \% \mathrm{D}_{2} \mathrm{O}$ at $\mathrm{pH} 8.0 . \mathrm{D}_{2} \mathrm{O}$ buffer was prepared the same way as DXP synthase exchange buffer except $99.9 \% \mathrm{D}_{2} \mathrm{O}$ was used to dissolve the buffer components. The samples were incubated at $25^{\circ} \mathrm{C}$ for 8,15 , and $40 \mathrm{~s}$ and $1,3,5,10$, and 30 $\mathrm{min}$, and then the reactions quenched by rapidly mixing with an equivalent of ice-cold quench buffer [trifluoroacetic acid and $3 \mathrm{M}$ guanidine hydrochloride (pH 1.4)] to acidify the final sample $\mathrm{pH}$ to 2.5 . The samples were immediately frozen in liquid nitrogen and stored at $-80{ }^{\circ} \mathrm{C}$ before analysis. The frozen deuterated sample was quickly thawed and loaded with an ice-cold syringe into a $20 \mu \mathrm{L}$ sample loop inside the refrigeration system. The protein sample ( $40 \mathrm{pmol})$ was carried by a $0.2 \mathrm{~mL} / \mathrm{min}$ digestion flow $(0.1 \%$ formic acid) into an immobilized pepsin column (Immobilized Pepsin Cartridge, $2.1 \mathrm{~mm} \times 30 \mathrm{~mm}$, Thermo Fisher) and digested at $15^{\circ} \mathrm{C}$ for $30 \mathrm{~s}$. The resultant peptides were immediately cooled to $0{ }^{\circ} \mathrm{C}$ through a heat exchanger and concentrated and desalted on a peptide trap (Optimize technology Peptide MacroTrap, $3 \mathrm{~mm} \times 8 \mathrm{~mm}$ ). The peptides were eluted and separated over 15 min through a reversed-phase C18 HPLC column (Agilent Poroshell $300 \mathrm{SB}-\mathrm{C} 18,2.1 \mathrm{~mm} \times 75 \mathrm{~mm}$ ) at a flow rate of $0.2 \mathrm{~mL} / \mathrm{min}$ with a $0{ }^{\circ} \mathrm{C} 2$ to $40 \%$ acetonitrile gradient containing $0.1 \%$ formic acid. ESI-Fourier transform mass spectrometry measurements began $5 \mathrm{~min}$ after the initiation of the elution process and lasted $10 \mathrm{~min}$. The time from initiation of digestion to elution of the last peptide was $<20$ min. Bruker Daltonics DataAnalysis 4.0 was used for spectrum analysis and data treatment. Peptides were identified from undeuterated samples by a customized program DXgest, which matches the experimental peptide mass with the theoretically generated peptic peptide mass by using statistical data for the pepsin cleavage pattern under HDX conditions. The mass tolerance was set at $1.0 \mathrm{ppm}$. The bimodal EX1 kinetics MS data were deconvoluted with HXExpress $2 .{ }^{43}$

\section{Limited Trypsinolysis of Wild Type DXP Synthase and H49 and H299 Variants.}

All trypsin digests were performed in an anaerobic chamber up to the point of quenching the trypsinolysis reaction. Wild type DXP synthase and variants $(9 \mu \mathrm{M})$ were preincubated in the presence or absence of MAP $\left(20 K_{\mathrm{i}}^{\mathrm{MAP}}, 65 \mu \mathrm{M}\right.$ for the wild type, $4.6 \mathrm{mM}$ for $\mathrm{H} 49 \mathrm{~A}$, and $4.9 \mathrm{mM}$ for $\mathrm{H} 299 \mathrm{~N}$ ) in DXP synthase reaction buffer (described above) for $45 \mathrm{~min}$ at $6{ }^{\circ} \mathrm{C}$. Trypsinolysis was initiated upon addition of trypsin $(4.5 \mathrm{ng} / \mu \mathrm{L}$, final volume of $80 \mu \mathrm{L})$. The resulting reaction mixture was incubated at $6{ }^{\circ} \mathrm{C}$, and aliquots $(10 \mu \mathrm{L})$ were quenched into $10 \mu \mathrm{L}$ of cold $2 \times$ sodium dodecyl sulfate (SDS) loading dye [100 mM 2-amino-2(hydroxymethyl)-1,3-propanediol (Tris) (pH 6.8), 4\% (w/v) SDS, $0.2 \%$ (w/v) bromophenol blue, $20 \%(\mathrm{w} / \mathrm{v})$ glycerol, and $200 \mathrm{mM}$ dithiothreitol] at the following times: $0.5,5,10,30$, 
60, and 150 min (Figure 6) or 0.5, 1, 2.5, 5, 7.5, and 10 min (Figure 7). The quenched solution was immediately vortexed and flash-frozen in liquid $\mathrm{N}_{2}$ to prevent further degradation. All samples were removed from the anaerobic chamber and incubated on dry ice until the time course was completed, at which point all samples were boiled at $100{ }^{\circ} \mathrm{C}$ for 5 min to denature proteins/peptides. Peptides in each sample $(15 \mu \mathrm{L})$ were separated by SDS-polyacrylamide gel electrophoresis (10\% gel) at room temperature, and gels were subsequently stained with ProtoBlue Safe colloidal coomassie stain (National Diagnostics). Bands of interest were excised and placed in doubly deionized $\mathrm{H}_{2} \mathrm{O}$ for further processing. Intact masses were determined by the Johns Hopkins Mass Spectrometry Core, and peptide sequences were identified by the Taplin Mass Spectrometry Facility at Harvard University.

\section{RESULTS}

\section{Steady State Characterization of H49 and H299 Variants.}

Previous kinetic analyses of $\mathrm{H} 49$ and $\mathrm{H} 299$ variants (and analogous variants on $D$. radiodurans DXP synthase) were conducted under aerobic conditions, ${ }^{19,21}$ prior to the discovery that oxygenase activity interferes in mechanistic and structural studies of DXP synthase. ${ }^{13,16}$ Here, we show that all $\mathrm{H} 49$ and $\mathrm{H} 299$ variants display significant, albeit inefficient, oxygenase activity (Figure S1); accordingly, we conducted detailed kinetic analyses under anaerobic conditions, to exclude any potentially confounding effects of oxidative pyruvate decarboxylation in comparing kinetic parameters for H49A, H49N, H299A, H299N, and wild type DXP synthase. Variants were selected to determine the effects of both conservative (asparagine) and nonconservative (alanine) substitutions on substrate affinity, catalytic activity, and inhibition under anaerobic conditions.

\section{Kinetic Characterization of DXP Formation.}

Kinetic parameters of these variants are comparable under anaerobic and aerobic conditions, which is expected given the low catalytic efficiency of DXP synthase-catalyzed acetate formation compared to that of DXP formation (Table 1, Table S1, and Figures S2 and S3). ${ }^{13}$ H49 DXP synthase variants display decreased catalytic efficiency with respect to both substrates in the presence or absence of oxygen, with substitution of either residue having more pronounced effects on $k_{\text {cat }} / K_{\mathrm{m}}{ }^{\text {pyruvate }}$ than on $k_{\text {cat }} / K_{\mathrm{m}}{ }^{\mathrm{D}-\mathrm{GAP}}$. H49 variants exhibit decreased $k_{\text {cat }} / K_{\mathrm{m}}$ pyruvate values compared to that of wild type DXP synthase (76-fold for H49A and 58-fold for $\mathrm{H} 49 \mathrm{~N}$ ) largely due to a $K_{\mathrm{m}}$ effect, indicating stabilizing interactions between $\mathrm{H} 49$ and pyruvate. Modest decreases in $k_{\text {cat }}$ are observed on H49A and H49N, suggesting that $\mathrm{H} 49$ is not critical for the rate-limiting step of LThDP formation.

Substitution at $\mathrm{H} 299$ results in an even greater decrease in $k_{\mathrm{cat}} / K_{\mathrm{m}}$ pyruvate relative to that of wild type DXP synthase (846-fold decrease for H299A and 185-fold decrease for H299N), arising from significant impacts on both $K_{\mathrm{m}}$ and $k_{\text {cat }}$ (Table 1 and Figure S3). This points to a key role for $\mathrm{H} 299$ in the rate-limiting LThDP formation step and binding of pyruvatederived intermediates along the reaction coordinate.

A comparison of kinetic parameters for nonconservative (Ala) and conservative (Asn) variants (Table 1 and Figure S3) indicates that Ala substitution has a greater impact on the catalytic efficiency with respect to pyruvate compared to Asn substitution, mainly due to a 
$k_{\text {cat }}$ effect. The ability of Asn to hydrogen bond does not completely restore activity compared to Ala variants, suggesting that the acid/base characteristics of His are important in catalysis.

\section{Characterization of DXP Synthase Inhibition by MAP.}

MAP, the pyruvate analogue inhibitor of DXP synthase, has been used to investigate the DXP synthase mechanism. ${ }^{23}$ MAP forms the LThDP-like intermediate PLThDP, which cannot undergo decarboxylation, and is thus a useful probe for interrogating steps prior to LThDP decarboxylation. ${ }^{24,25}$ To provide biochemical evidence of the roles of H49 and H299 in MAP binding, suggested by the PLThDP-bound structure of DXP synthase,,${ }^{16}$ we determined the effects of substituting $\mathrm{H} 49$ and $\mathrm{H} 299$ on $K_{\mathrm{i}}^{\mathrm{MAP}}$ (Table 2 and Figure S4). Because of the lower activity of the variants investigated in this study, the condition [DXP synthase $] \ll[\mathrm{MAP}]$ could not be met; thus, the Morrison equation was utilized to calculate $K_{\mathrm{i}}^{\mathrm{MAP}}$, and the mode of inhibition was determined by comparison of $\mathrm{IC}_{50}{ }^{\mathrm{MAP}}$ at $2 K_{\mathrm{m}}$ pyruvate and $10 K_{\mathrm{m}}$ pyruvate ${ }^{26}$ As expected, MAP is competitive with respect to pyruvate in all cases, indicated by an increase in $\mathrm{IC}_{50}{ }^{\mathrm{MAP}}$ with an increase in pyruvate concentration (Table 2 and Figure S4). Consistent with the effects of these substitutions on $K_{\mathrm{m}}{ }^{\text {pyruvate }}$, a significant increase in $K_{\mathrm{i}}^{\mathrm{MAP}}$ is observed on all variants compared to wild type DXP synthase with the most dramatic increase in $K_{\mathrm{i}}^{\mathrm{MAP}}$ observed on H299A (Table 2). These results provide additional evidence that $\mathrm{H} 49$ and $\mathrm{H} 299$ make important interactions with MAP, and by extension the donor substrate, to facilitate predecarboxylation intermediate formation.

\section{Impact of Substitution at H49 and H299 on the Circular Dichroism (CD) Profile.}

Given the proximities of $\mathrm{H} 49$ and $\mathrm{H} 299$ to EX1 regions identified by HDX-MS, ${ }^{18}$ their interactions with LThDP identified in structural studies, ${ }^{16}$ and the effects of their substitution on kinetic parameters, ${ }^{19,21}$ we hypothesized that these residues aid in coordinating a closed conformation that promotes LThDP formation and stabilization. DXP synthase with bound ThDP or ThDP adducts can be studied by CD. ${ }^{12,27-32}$ In the presence of a saturating ThDP concentration, DXP synthase is characterized by a $\lambda_{\min }$ between 320 and $330 \mathrm{~nm}$ corresponding to the $4^{\prime}$-aminopyrimidine (AP) tautomer of ThDP [E-ThDP (Figure 1)]. 12,28-30 Tetrahedral ThDP intermediates such as LThDP and C2 $a$-hydroxyethylThDP (HEThDP) exist in the $1^{\prime}, 4^{\prime}$-iminopyrimidine (IP) tautomer in enzyme active sites [ELThDP (Figure 1)] and are characterized by a $\lambda_{\max }$ between 300 and $314 \mathrm{~nm} .{ }^{12,27-32}$ The optical properties of enzyme-bound ThDP intermediates are sensitive to the active site environment. ${ }^{27-30,32}$ Given the significant structural changes revealed in the active site environment in open and closed conformations, which we hypothesize involves both H49 and H299, we reasoned that changes in active site architecture on DXP synthase variants may manifest in changes in CD signatures for the AP and IP forms. Thus, we investigated the effects of substitution at $\mathrm{H} 49$ and $\mathrm{H} 299$ on the steady state CD signatures of AP and IP forms on DXP synthase.

\section{Investigation of the AP Tautomer of ThDP on DXP Synthase Variants.}

In the absence of substrates, ThDP-bound wild type DXP synthase displays an AP CD signature with an average $\lambda_{\min }$ at $320 \mathrm{~nm}$ (Figure 3 ). However, close inspection of the CD 
spectra of $\mathrm{H} 49$ and $\mathrm{H} 299$ variants obtained in the presence of saturating ThDP concentrations reveals a shorter $\lambda_{\min }(301-311 \mathrm{~nm})$ for the AP tautomer on these variants compared to that of the wild type (Figure 3 ). The AP signal intensity is proposed to arise from a charge transfer between the aminopyrimidine and thiazolium rings, and the nature of this signal depends on the active site environment; ${ }^{28-30}$ therefore, it is plausible that the shift in the AP signal on H49 and H299 variants signifies changes in the active site architecture compared to that of the wild type enzyme.

\section{Investigation of the IP Tautomer of ThDP on DXP Synthase Variants.}

Under anaerobic conditions, accumulation of LThDP on DXP synthase is apparent in the buildup of a CD signal $\left(\lambda_{\max }=298-300 \mathrm{~nm}\right.$ ) upon addition of pyruvate to ThDP-bound DXP synthase. ${ }^{13}$ Similar to the case for the wild type enzyme, accumulation of a positive CD signal is observed on H49 and H299 DXP synthase variants. However, the magnitude of the increase in the intensity of this signal is smaller for variants and depends on the substitution, with the extent of signal intensity buildup on H49N being greater than on H49A (Figure 4A-C). A similar observation is made for H299N compared to H299A (Figure 4DF). It is notable that this trend parallels $k_{\text {cat }}$ effects of Asn versus Ala substitution, suggesting they may be correlated. It is possible that the lower signal intensity of LThDP on variants compared to the wild type enzyme could indicate a decrease in the amount of LThDP accumulation and/or a change in the molar ellipticity of LThDP in the altered active sites of the variants. Overall, changes in the CD signatures of ThDP- and LThDP-bound DXP synthase variants compared to wild type DXP synthase support a role for H49 and H299 in maintaining the wild type active site architecture required for LThDP formation.

\section{Roles of H49 and H299 in Conformational Dynamics.}

Given that LThDP formation is accompanied by a shift in the conformational equilibrium to a closed form, ${ }^{16,18}$ and the observation that substitution of $\mathrm{H} 49$ or $\mathrm{H} 299$ impedes LThDP formation, it follows that $\mathrm{H} 49$ and $\mathrm{H} 299$ variants may display different conformational dynamics relative to that of the wild type enzyme. Thus, we investigated the conformational dynamics of select variants by two methods, HDX-MS and limited trypsinolysis.

Importantly, the global secondary structure and stability of DXP synthase are unaffected by substitutions at $\mathrm{H} 49$ and $\mathrm{H} 299$ (Figure S5), indicating that the structural changes described here are local effects of these substitutions.

\section{Conformational Equilibrium of Histidine Variants Detected by HDX-MS.}

As noted, a previous investigation of wild type E. coli DXP synthase indicated that three regions near the active site (residues 42-56, 183-199, and 278-298) exhibit EX1 exchange kinetics. ${ }^{18}$ Evident from the structures of closed and open $D$. radiodurans DXP synthase, ${ }^{16}$ the EX1 regions become exposed to solvent in the open conformation, and thus more readily undergo H-D exchange. Therefore, the closed-to-open conformational change represents an H-D exchange "unfolding" transition. The mass spectra of these regions are characterized by a bimodal distribution indicating two populations: a higher- $m / z$ envelope that has undergone an unfolding event and, thus, greater $\mathrm{H}-\mathrm{D}$ exchange and a lower- $\mathrm{m} / \mathrm{z}$ envelope, which has not undergone significant unfolding or exchange. The relative abundance of these envelopes depends upon the ligand state of DXP synthase. ${ }^{18}$ In the presence of MAP, this 
equilibrium shifts toward the lower- $m / z$ population indicating a closed state, whereas binding of D-GAP or DXP shifts the equilibrium toward an open conformation represented by a higher- $m / z$ envelope. These changes in conformational equilibria are quantitatively described as rates of unfolding $(k)$ or half-lives of unfolding $\left(t_{1 / 2}\right)$. Conditions that shift the conformational equilibrium of DXP synthase toward the open conformation, such as the presence of D-GAP, are indicated by a faster rate of unfolding compared to the control to expose EX1 regions to solvent. In contrast, the closed conformation that occurs in the presence of MAP in which EX1 regions are protected from H-D exchange is characterized by a slower rate of unfolding (longer half-life of unfolding). The unfolding transitions observed by HDX-MS are supported by the pre- and postdecarboxylation structures of $D$. radiodurans DXP synthase that illustrate an increase in the extent of solvent exposure at all three EX1 regions in the open state compared to the closed state. ${ }^{16}$ Importantly, the movement of the fork (including H299) and spoon motifs away from the active site results in the change in solvent accessibility of the EX1 regions.

We utilized HDX-MS to quantify and compare the effects of substitution at H49 and H299 on the conformational dynamics of the three EX1 regions of DXP synthase. Here, we monitored $\mathrm{H}-\mathrm{D}$ exchange on H49A and $\mathrm{H} 299 \mathrm{~N}$ (the most soluble variants) in the absence of ligands (Figure 5, Figures S6-S8, and Table 3). Examination of the three peptides displaying EX1 kinetics indicates H49A and H299N are characterized by faster exchange rates in all three regions compared to that of the wild type enzyme (Table 3). Given that the global structure of DXP synthase is unaffected by substitution at H49 and H299 (Figure S5), it is likely that the increase in the level of exchange is due to local unfolding (closed-to-open transition) that exposes the EX1 peptide regions to solvent. For example, within $8 \mathrm{~s}, 74 \%$ and $49 \%$ of peptide 51-58 in H49A and H299N (Figure 5), respectively, have undergone an unfolding transition compared to only $15 \%$ in the wild type (control). This correlates to 150 fold (H49A) and 55-fold (H299N) decreases in the $t_{1 / 2}$ of unfolding compared to that of wild type DXP synthase (Table 3). A similar trend is observed across all three EX1 regions (Table 3 and Figures S6-S8) on both variants, indicating that changes in conformational dynamics caused by substitution at $\mathrm{H} 49$ and $\mathrm{H} 299$ simultaneously influence all three regions. Overall, these results suggest that substitution at $\mathrm{H} 49$ and $\mathrm{H} 299$ shifts the conformational equilibrium of ThDP-bound DXP synthase toward an open conformation compared to the conformational equilibrium of the wild type enzyme under the same conditions. This is consistent with changes in the CD profiles of ThDP-bound DXP synthase variants that are characterized by a shorter $\lambda_{\min }$ compared to that of wild type ThDP-bound DXP synthase (described above), further supporting the hypothesis that these residues are important for maintaining the active site architecture in a closed conformation.

Notably, EX1 hydrogen-deuterium exchange kinetics of the three peptide regions of H49A and $\mathrm{H} 299 \mathrm{~N}$ are observed over a significantly shorter time period (Figure 5 and Figures S6S8) compared to the previous study of wild type DXP synthase. ${ }^{18}$ Because these variants adopt a more open conformation, H-D exchange occurs quickly in the three EX1 regions of interest; thus, shorter time points were necessary for this study. All three peptides were fully deuterated within 3 min on both variants (Figure 5 and Figures S6-S8). To fully illustrate the characteristic EX1 kinetics of these three peptides, H-D exchange was quickly quenched 
within 8-60 s, enabling the capture of changes in abundance of folded and unfolded populations of $\mathrm{H} 49 \mathrm{~A}$ and $\mathrm{H} 299 \mathrm{~N}$ over time.

\section{Histidine Variants Display Increased Susceptibility to Limited Trypsinolysis in Flexible Regions near the Active Site.}

Protein conformational changes can be monitored using limited proteolysis, which reports on changes in the proteolytic susceptibility of flexible regions in a protein. ${ }^{33,34}$ As noted, changes in the conformational equilibrium of H49A and H299N DXP synthase compared to that of the wild type enzyme are observed by HDX-MS. However, because the variants adopt an open conformation, the level of solvent exposure of the three EX1 peptides is high; backbone hydrogen-deuterium exchange occurs quickly on these peptides and is challenging to capture. Both fork and spoon regions include potential trypsin digest sites, thus, limited trypsinolysis for monitoring coarse changes in the conformation of solvent-exposed regions of DXP synthase in the presence or absence of MAP on wild type, H49A, and H299N DXP synthase.

Limited trypsinolysis of wild type DXP synthase in the absence of ligands reveals two major cleavage products of 44 and $34 \mathrm{kDa}$ in addition to the full length enzyme at $68 \mathrm{kDa}$ (Figure $6 \mathrm{~A})$. Mass spectrometry sequencing of cleavage products indicates that cleavage at K223 forms the $44 \mathrm{kDa}$ peptide and cleavage at K313 forms the $34 \mathrm{kDa}$ peptide. Interestingly, $\mathrm{K} 313$ is within the spoon motif that undergoes a dramatic shift in the transition from the closed state to the open state (Figure 2) ${ }^{18}$ Thus, we might expect to observe an effect of MAP binding on trypsinolysis in this region. Indeed, comparison of the trypsinolysis profile of wild type DXP synthase with or without MAP reveals accumulation of the $44 \mathrm{kDa}$ peptide in the presence of MAP (Figure 6A). These results indicate that in the presence of PLThDP, K313 is protected from typsinolysis, implying that the conformation of this region changes in response to donor ligand binding and activation. This protection from trypsinolysis parallels the effect of MAP on the solvent accessibility of DXP synthase in HDX-MS and structural studies, ${ }^{16,18}$ suggesting the closed conformation is proteolyzed to the $44 \mathrm{kDa}$ peptide.

As illustrated in Figure 6D, the K313-containing, solvent-exposed spoon motif that includes H299 is positioned in front of the peptide, which includes H49. Substitution at H49 and H299 influences the trypsin digest profile of DXP synthase in the presence or absence of MAP (Figure 6A-C). Both H49A and H299N display increased susceptibility to trypsinolysis at K313 compared to wild type DXP synthase, which is indicated by accumulation of the $34 \mathrm{kDa}$ peptide in the case of H49A and H299N (Figure 6A-C). The increased level of accumulation of other minor cleavage products is also apparent in the H49A and H299N digest profiles compared to the wild type, implying an increased level of trypsinolysis occurs in other regions of the protein, as well. These results indicate that substitution of H49 or H299 impacts the conformation of DXP synthase, consistent with HDX-MS and structural studies suggesting a shifting of the conformational equilibrium to a more open state. Preincubation of H49A and H299N with a saturating MAP concentration, a condition known to induce a closed conformation and promote sustained accumulation of the $44 \mathrm{kDa}$ peptide under limited trypsinolysis on wild type DXP synthase, does not have the 
same pronounced effect on the variants; modest accumulation of the $44 \mathrm{kDa}$ peptide in the digest profiles of $\mathrm{H} 49 \mathrm{~A}$ and $\mathrm{H} 299 \mathrm{~N}$ in the presence of MAP is apparent only over a short time period from 0.5 to $10 \mathrm{~min}$ (Figure 7). Unlike the digest profile of the wild type enzyme in the presence of MAP, buildup of the $44 \mathrm{kDa}$ peptide is not sustained beyond $10 \mathrm{~min}$ with H49A and H299N (Figure 6A-C). These results suggest that MAP-bound DXP synthase is more susceptible to trypsinolysis at K313 when the interaction of PLThDP with H49 or H299 is interrupted. The effects of these substitutions on the proteolytic susceptibility of DXP synthase provide structural evidence that both residues are important for coordinating a conformational change that affects the solvent accessibility of the loop bearing K313.

\section{DISCUSSION}

We hypothesized that E. coli DXP synthase active site residues H49 and H299 (H51 and H304 on D. radiodurans DXP synthase) play dual roles in catalysis and conformational dynamics. This study applied a combination of biochemical and structural methods to study the effects of substituting $\mathrm{H} 49$ and $\mathrm{H} 299$ on the conformational equilibrium and catalytic efficiency, to establish the first direct link between enzymatic determinants of chemistry and conformation. Results from detailed kinetic analysis of H49 and H299 variants confirm the roles of $\mathrm{H} 49$ and $\mathrm{H} 299$ in binding of pyruvate and MAP and are consistent with a model in which the positively charged histidines are required to stabilize interactions with the carboxyl and phosphonyl groups of LThDP and PLThDP. In addition, H299 was found to be critical for catalysis in the rate-limiting step, LThDP formation, whereas H49 may not be absolutely required. Importantly, our results indicate that substitution of either H49 or H299 also alters the conformational equilibrium of DXP synthase. Using limited trypsinolysis and HDX-MS, methods that report on different peptide regions near the active site, we found that substitution of either residue results in a dramatic shift to an open conformation. These results support a model in which these active site histidines, known to be critical for substrate binding and catalysis, are also involved in stabilizing the closed conformation in the presence or absence of a ligand.

Limited trypsinolysis emerged as a useful tool for studying DXP synthase conformational dynamics, revealing ligand- and enzyme-dependent changes in the susceptibility of a solvent-exposed region of the spoon motif to proteolytic cleavage by trypsin at K313. This region is readily cleaved in the absence of ligands on wild type DXP synthase but is protected from trypsinolysis in the presence of MAP, consistent with the MAP-induced shift to a closed conformation detected by HDX-MS. ${ }^{18}$ Thus, limited proteolysis provides an accessible method for detecting open and closed conformations of DXP synthase. The loop containing K313, subsequently determined to reside within the spoon motif, was not detected as a ligand-responsive dynamic region by HDX-MS, likely due to a high degree of solvent exposure that leads to rapid deuteration. Changes in loop conformation that do not alter the backbone solvent accessibility but do alter the accessibility to trypsin cannot be studied by HDX-MS but are easily detected by limited proteolysis methods. Applying this method to the study of $\mathrm{H} 49$ and $\mathrm{H} 299$ variants, we demonstrated more rapid cleavage at K313 compared to wild type DXP synthase, indicating a shift to the open conformation that exposes K313 to the trypsin active site, and supporting the roles of H49 and H299 in coordinating a closed conformation. In contrast to its effect on wild type DXP synthase, 
addition of MAP has modest effects on the rapid cleavage profile observed for H49 and H299 variants, consistent with their inability to effectively stabilize PLThDP in a closed conformation.

As predicted, we observed shifts in the $\mathrm{CD} \lambda_{\min }$ of the AP forms and reduced amplitudes of CD signals assigned to LThDP with the loss of $\mathrm{H} 49$ or H299, consistent with altered active site environments on these variants. Lower CD signal amplitudes could signify differences in the molar ellipticities of LThDP bound to variants with altered active site environments. It is also possible that these substitutions alter the ability of DXP synthase to stabilize LThDP, perhaps lowering the barrier to decarboxylation that when combined with slower LThDP formation leads to lower steady state concentrations of LThDP. Finally, it is possible that the observed CD signal represents a fraction of LThDP-bound active sites that are present in a conformation that produces a CD signal consistent with a classical LThDP signal. If the closed conformation of LThDP-bound DXP synthase is required to detect the IP form by $\mathrm{CD}$, the change in signal amplitude on His variants could reflect the shift in the conformational equilibrium to the open form, resulting in a smaller population of the closed conformation and a lower signal intensity.

In summary, our results demonstrate the direct effect of altering conformational dynamics on catalysis and, together with crystallographic snapshots of closed and open states, provide key insights into the coordination of conformation and chemistry. We have established that H299 is required to promote the closed conformation, and its removal facilitates an open conformation. These observations are supported by recent structural studies showing that $D r H 304$ interacts with the phosphonyl group of PLThDP in the closed conformation, whereas this residue is removed from the active site in the open conformation. ${ }^{19}$ Interestingly, E1-PDH H407, equivalent to H299 on DXP synthase, is part of a critical dynamic loop, which changes conformation along the PDH reaction coordinate. ${ }^{35,36} \mathrm{In}$ contrast to $\mathrm{H} 407$ on E1-PDH, which is important in steps following decarboxylation, movement of $\mathrm{H} 299$ out of the active site seems to play a role in destabilizing LThDP to induce decarboxylation, suggesting $\mathrm{H} 299$ may have evolved to play a different role to support the unique mechanism of DXP synthase. We have also established a similar requirement for $\mathrm{H} 49$ in the conformational dynamics of DXP synthase; however, it remains unclear how H49 stabilizes the closed conformation and how H49 and H299 may contribute to anchoring the spoon and fork motifs in the closed conformation. The peptide that includes H49 is relatively less flexible compared to the fork and spoon motifs that include H299; thus, it is possible that interaction of $\mathrm{H} 49$ and $\mathrm{H} 299$, either directly or indirectly through a network, is necessary to pin down the fork and spoon motifs in the closed conformation to ensure that both $\mathrm{H} 49$ and $\mathrm{H} 299$ interact with and stabilize the predecarboxylation intermediate. Interrogating the structures of these histidine variants through X-ray crystallography should shed light on the mechanism by which H49 and H299 coordinate the closed conformation.

The detailed mechanism by which D-GAP induces LThDP decarboxylation remains elusive. The results of this study suggest that binding of D-GAP could alter an active site network, which in turn disrupts stabilizing interactions of H49 and H299 with LThDP. As supported by the study presented here, interruption of these interactions among $\mathrm{H} 49, \mathrm{H} 299$, and 
LThDP could induce an open conformation, leading to destabilization of LThDP, which facilities decarboxylation. H49- and H299-dependent coordination of the closed-to-open equilibrium could also be critical for other proposed mechanisms of LThDP decarboxylation. For example, LThDP decarboxylation may be reversible ${ }^{37,38}$ such that LThDP and the enamine and $\mathrm{CO}_{2}$ exist in an equilibrium in the closed conformation, which prevents the release of $\mathrm{CO}_{2}$ from the enzyme. In this case, a transition state that includes a tight network of interactions among $\mathrm{H} 49, \mathrm{H} 299$, and $\mathrm{CO}_{2}$ in the closed conformation could sequester $\mathrm{CO}_{2}$ in the active site. ${ }^{39}$ Subsequent binding of D-GAP could induce an open conformation, relieving these enzyme-substrate interactions to liberate $\mathrm{CO}_{2},{ }^{39}$ ultimately driving the equilibrium toward the enamine. Additional structural and biochemical investigation is required to understand the detailed mechanism of D-GAP-induced LThDP decarboxylation on DXP synthase. Continued investigation of the distinct conformational dynamics of DXP synthase is essential to guide the rational design of selective inhibitors of this anti-infective agent target.

\section{Supplementary Material}

Refer to Web version on PubMed Central for supplementary material.

\section{ACKNOWLEDGMENTS}

The authors thank Katie Tripp and the Johns Hopkins University Center for Molecular Biophysics for access to CD spectrometers and technical assistance. The authors gratefully acknowledge Ross Tomaino (Taplin Mass Spectrometry Center at Harvard Medical School) and Robert Cole (Johns Hopkins Mass Spectrometry and Proteomics Facility) for their assistance with limited trypsinolysis peptide sequencing.

Funding

This work was supported by National Institutes of Health Grant GM084998 to C.L.F.M., F.J., A.A.D., and K.L.H.; T32GM008763 for A.A.D. and T32GM08018901 for K.L.H.

\section{ABBREVIATIONS}

$\begin{array}{ll}\text { DXP } & \text { 1-deoxy-D-xylulose 5-phosphate } \\ \text { ThDP } & \text { thiamin diphosphate } \\ \text { D-GAP } & \text { D-glyceraldehyde 3-phosphate } \\ \text { PLP } & \text { pyridoxal phosphate } \\ \text { TK } & \text { transketolase } \\ \text { E1-PDH } & \text { E1 component of the E. coli pyruvate dehydrogenase complex } \\ \text { LThDP } & \text { C2 } a \text {-lactylthiamin diphosphate } \\ \text { HDX-MS } & \text { hydrogen-deuterium exchange mass spectrometry } \\ \text { MAP } & \text { methylacetylphosphonate } \\ \text { PLThDP } & \text { C2 } a \text {-phosphonolactylthiamin diphosphate }\end{array}$


HEThDP C2a-hydroxyethylThDP
AP
$4^{\prime}$-aminopyrimidine tautomer of ThDP
IP
$1^{\prime}, 4^{\prime}$-iminopyrimidine tautomer of ThDP

\section{REFERENCES}

(1). Du Q, Wang H, and Xie J (2011) Thiamin (vitamin B1) biosynthesis and regulation: a rich source of antimicrobial drug targets? Int. J. Biol. Sci 7, 41-52. [PubMed: 21234302]

(2). Mukherjee T, Hanes J, Tews I, Ealick SE, and Begley TP (2011) Pyridoxal phosphate: biosynthesis and catabolism. Biochim. Biophys. Acta, Proteins Proteomics 1814, 1585-1596.

(3). Sprenger GA, Schorken U, Wiegert T, Grolle S, de Graaf AA, Taylor SV, Begley TP, BringerMeyer S, and Sahm H (1997) Identification of a thiamin-dependent synthase in Escherichia coli required for the formation of the 1-deoxy-D-xylulose 5-phosphate precursor to isoprenoids, thiamin, and pyridoxol. Proc. Natl. Acad. Sci. U. S. A 94, 12857-12862. [PubMed: 9371765]

(4). Lois LM, Campos N, Putra SR, Danielsen K, Rohmer M, and Boronat A (1998) Cloning and characterization of a gene from Escherichia coli encoding a transketolase-like enzyme that catalyzes the synthesis of D-1-deoxyxylulose 5-phosphate, a common precursor for isoprenoid, thiamin, and pyridoxol biosynthesis. Proc. Natl. Acad. Sci. U. S. A 95, 2105-2110. [PubMed: 9482846]

(5). Bartee D, and Freel Meyers CL (2018) Targeting the Unique Mechanism of Bacterial 1-Deoxy-dxylulose-5-phosphate Synthase. Biochemistry 57, 4349-4356. [PubMed: 29944345]

(6). Bartee D, and Freel Meyers CL(2018) Toward Understanding the Chemistry and Biology of 1Deoxy-d-xylulose 5-Phosphate (DXP) Synthase: A Unique Antimicrobial Target at the Heart of Bacterial Metabolism. Acc. Chem. Res. 51, 2546-2555. [PubMed: 30203647]

(7). Smith JM, Vierling RJ, and Meyers CF (2012) Selective inhibition of E. coli 1-deoxy-Dxylulose-5-phosphate synthase by acetylphosphonates(). MedChemComm 3, 65-67. [PubMed: 23326631]

(8). Smith JM, Warrington NV, Vierling RJ, Kuhn ML, Anderson WF, Koppisch AT, and Freel Meyers CL (2014) Targeting DXP synthase in human pathogens: enzyme inhibition and antimicrobial activity of butylacetylphosphonate. J. Antibiot. 67, 77-83. [PubMed: 24169798]

(9). Sanders S, Vierling RJ, Bartee D, DeColli AA, Harrison MJ, Aklinski JL, Koppisch AT, and Freel Meyers CL (2017) Challenges and Hallmarks of Establishing Alkylacetylphosphonates as Probes of Bacterial 1-Deoxy-d-xylulose 5-Phosphate Synthase. ACS Infect. Dis 3, 467-478. [PubMed: 28636325]

(10). Xiang S, Usunow G, Lange G, Busch M, and Tong L (2007) Crystal structure of 1-deoxy-Dxylulose 5-phosphate synthase, a crucial enzyme for isoprenoids biosynthesis. J. Biol. Chem 282, 2676-2682. [PubMed: 17135236]

(11). Morris F, Vierling R, Boucher L, Bosch J, and Freel Meyers CL (2013) DXP synthase-catalyzed $\mathrm{C}-\mathrm{N}$ bond formation: nitroso substrate specificity studies guide selective inhibitor design. ChemBioChem 14, 1309-1315.

(12). Patel H, Nemeria NS, Brammer LA, Freel Meyers CL, and Jordan F (2012) Observation of thiamin-bound intermediates and microscopic rate constants for their interconversion on 1-deoxyD-xylulose 5-phosphate synthase: 600-fold rate acceleration of pyruvate decarboxylation by Dglyceraldehyde-3-phosphate. J. Am. Chem. Soc 134, 18374-18379. [PubMed: 23072514]

(13). DeColli AA, Nemeria NS, Majumdar A, Gerfen GJ, Jordan F, and Freel Meyers CL (2018) Oxidative decarboxylation of pyruvate by 1-deoxy-d-xyulose 5-phosphate synthase, a central metabolic enzyme in bacteria. J. Biol. Chem 293, 10857-10869. [PubMed: 29784878]

(14). Eubanks LM, and Poulter CD (2003) Rhodobacter capsulatus 1-deoxy-D-xylulose 5-phosphate synthase: steady-state kinetics and substrate binding. Biochemistry 42, 1140-1149. [PubMed: 12549936] 
(15). Brammer LA, Smith JM, Wade H, and Meyers CF (2011) 1-Deoxy-D-xylulose 5-phosphate synthase catalyzes a novel random sequential mechanism. J. Biol. Chem 286, 36522-36531. [PubMed: 21878632]

(16). Chen PY, DeColli AA, Freel Meyers CL, and Drennan CL (2019) X-ray crystallography-based structural elucidation of enzyme-bound intermediates along the 1-deoxy-D-xylulose 5-phosphate synthase reaction coordinate. J. Biol. Chem 294 (33), 12405-12414. [PubMed: 31239351]

(17). Patel H, Nemeria NS, Brammer LA, Freel Meyers CL, and Jordan F (2012) Observation of thiamin-bound intermediates and microscopic rate constants for their interconversion on 1-deoxyD-xylulose 5-phosphate synthase: 600-fold rate acceleration of pyruvate decarboxylation by Dglyceraldehyde-3-phosphate. J. Am. Chem. Soc. 134, 18374-18379. [PubMed: 23072514]

(18). Zhou J, Yang L, DeColli A, Freel Meyers C, Nemeria NS, and Jordan F (2017) Conformational dynamics of 1-deoxy-D-xylulose 5-phosphate synthase on ligand binding revealed by H/D exchange MS. Proc. Natl. Acad. Sci. U. S. A 114, 9355-9360. [PubMed: 28808005]

(19). Handa S, Dempsey DR, Ramamoorthy D, Cook N, Guida WC, Spradling TJ, White JK, Woodcock HL, and Merkler DJ (2018) Mechanistic Studies of 1-Deoxy-D-Xylulose-5-Phosphate Synthase from Deinococcus radiodurans. Zhongguo Shengwu Huaxue Yu Fenzi Shengwu Xuebao 4, 2.

(20). Battistini MR, Shoji C, Handa S, Breydo L, and Merkler DJ (2016) Mechanistic binding insights for 1-deoxy-D-xylulose-5-phosphate synthase, the enzyme catalyzing the first reaction of isoprenoid biosynthesis in the malaria-causing protists, Plasmodium falciparum and Plasmodium vivax. Protein Expression Purif. 120, 16-27.

(21). Querol-Audí J, Boronat A, Centelles JJ, and Imperial S (2014) Catalytically Important Residues in E. coli 1-Deoxy-D-Xylulose 5-Phosphate Synthase. J. Biosci. Med 2, 30-35.

(22). Querol J, Rodriguez-Concepcion M, Boronat A, and Imperial S (2001) Essential role of residue H49 for activity of Escherichia coli 1-deoxy-D-xylulose 5-phosphate synthase, the enzyme catalyzing the first step of the 2-C-methyl-D-erythritol 4-phosphate pathway for isoprenoid Synthesis. Biochem. Biophys. Res. Commun 289, 155-160. [PubMed: 11708793]

(23). Brammer LA, Smith JM, Wade H, and Meyers CF (2011) 1-Deoxy-D-xylulose 5-phosphate synthase catalyzes a novel random sequential mechanism. J. Biol. Chem 286, 36522-36531. [PubMed: 21878632]

(24). O'Brien TA, Kluger R, Pike DC, and Gennis RB (1980) Phosphonate analogues of pyruvate. Probes of substrate binding to pyruvate oxidase and other thiamin pyrophosphate-dependent decarboxylases. Biochim. Biophys. Acta 613, 10-17. [PubMed: 6990987]

(25). Arjunan P, Sax M, Brunskill A, Chandrasekhar K, Nemeria N, Zhang S, Jordan F, and Furey W (2006) A thiamin-bound, predecarboxylation reaction intermediate analogue in the pyruvate dehydrogenase E1 subunit induces large scale disorder-to-order transformations in the enzyme and reveals novel structural features in the covalently bound adduct. J. Biol. Chem 281, 1529615303. [PubMed: 16531404]

(26). Copeland RA (2005) Evaluation of enzyme inhibitors in drug discovery. A guide for medicinal chemists and pharmacologists. Methods Biochem. Anal 46, 1-265. [PubMed: 16350889]

(27). Zhang S, Liu M, Yan Y, Zhang Z, and Jordan F (2004) C2-alpha-lactylthiamin diphosphate is an intermediate on the pathway of thiamin diphosphate-dependent pyruvate decarboxylation. Evidence on enzymes and models. J. Biol. Chem 279, 54312-54318. [PubMed: 15501823]

(28). Jordan F, and Nemeria NS (2005) Experimental observation of thiamin diphosphate-bound intermediates on enzymes and mechanistic information derived from these observations. Bioorg. Chem 33, 190-215. [PubMed: 15888311]

(29). Patel H, Nemeria NS, Andrews FH, McLeish MJ, and Jordan F (2014) Identification of charge transfer transitions related to thiamin-bound intermediates on enzymes provides a plethora of signatures useful in mechanistic studies. Biochemistry 53, 2145-2152. [PubMed: 24628377]

(30). Jordan F, and Nemeria NS (2014) Progress in the experimental observation of thiamin diphosphate-bound intermediates on enzymes and mechanistic information derived from these observations. Bioorg. Chem 57, 251-262. [PubMed: 25228115] 
(31). Jordan F, Zhang Z, and Sergienko E (2002) Spectroscopic evidence for participation of the $1^{\prime}, 4^{\prime}$ imino tautomer of thiamin diphosphate in catalysis by yeast pyruvate decarboxylase. Bioorg. Chem 30, 188-198. [PubMed: 12406703]

(32). Nemeria N, Baykal A, Joseph E, Zhang S, Yan Y, Furey W, and Jordan F (2004) Tetrahedral intermediates in thiamin diphosphate-dependent decarboxylations exist as a $1^{\prime}, 4^{\prime}$-imino tautomeric form of the coenzyme, unlike the michaelis complex or the free coenzyme. Biochemistry 43, 6565-6575. [PubMed: 15157089]

(33). Fung BK, and Nash CR (1983) Characterization of transducin from bovine retinal rod outer segments. II. Evidence for distinct binding sites and conformational changes revealed by limited proteolysis with trypsin. J. Biol. Chem 258, 10503-10510. [PubMed: 6136510]

(34). Hubbard SJ, Eisenmenger F, and Thornton JM (1994) Modeling studies of the change in conformation required for cleavage of limited proteolytic sites. Protein Sci. 3, 757-768. [PubMed: 7520312]

(35). Kale S, Arjunan P, Furey W, andJordan F (2007) A dynamic loop at the active center of the Escherichia coli pyruvate dehydrogenase complex E1 component modulates substrate utilization and chemical communication with the E2 component. J. Biol. Chem 282, 28106-28116. [PubMed: 17635929]

(36). Arjunan P, Sax M, Brunskill A, Chandrasekhar K, Nemeria N, Zhang S, Jordan F, and Furey W (2006) A thiamin-bound, predecarboxylation reaction intermediate analogue in the pyruvate dehydrogenase E1 subunit induces large scale disorder-to-order transformations in the enzyme and reveals novel structural features in the covalently bound adduct. J. Biol. Chem 281, 1529615303. [PubMed: 16531404]

(37). Kluger R (2015) Decarboxylation, CO2 and the reversion problem. Acc. Chem. Res 48, 28432849. [PubMed: 26528892]

(38). Kluger R, and Tittmann K (2008) Thiamin diphosphate catalysis: enzymic and nonenzymic covalent intermediates. Chem. Rev 108, 1797-1833. [PubMed: 18491870]

(39). Goryanova B, Amyes TL, and Richard JP (2019) Role of the Carboxylate in Enzyme-Catalyzed Decarboxylation of Orotidine 5'-Monophosphate: Transition State Stabilization Dominates Over Ground State Destabilization. J. Am. Chem. Soc 141, 13468-13478. [PubMed: 31365243]

(40). Nemeria NS, Shome B, DeColli AA, Heflin K, Begley TP, Meyers CF, and Jordan F (2016) Competence of Thiamin Diphosphate-Dependent Enzymes with 2'-Methoxythiamin Diphosphate Derived from Bacimethrin, a Naturally Occurring Thiamin Antivitamin. Biochemistry 55, 11351148. [PubMed: 26813608]

(41). Brammer LA (2012) Toward investigating 1-deoxy-D-xylulose-5-phosphate synthase as a newanti-infective target. Ph.D. Thesis, The Johns Hopkins University, Baltimore.

(42). Morrison JF (1969) Kinetics of the reversible inhibition of enzyme-catalysed reactions by tightbinding inhibitors. Biochim. Biophys. Acta 185, 269-286. [PubMed: 4980133]

(43). Guttman M, Weis DD, Engen JR, and Lee KK (2013) Analysis of overlapped and noisy hydrogen/deuterium exchange mass spectra. J. Am. Soc. Mass Spectrom 24, 1906-1912. [PubMed: 24018862] 


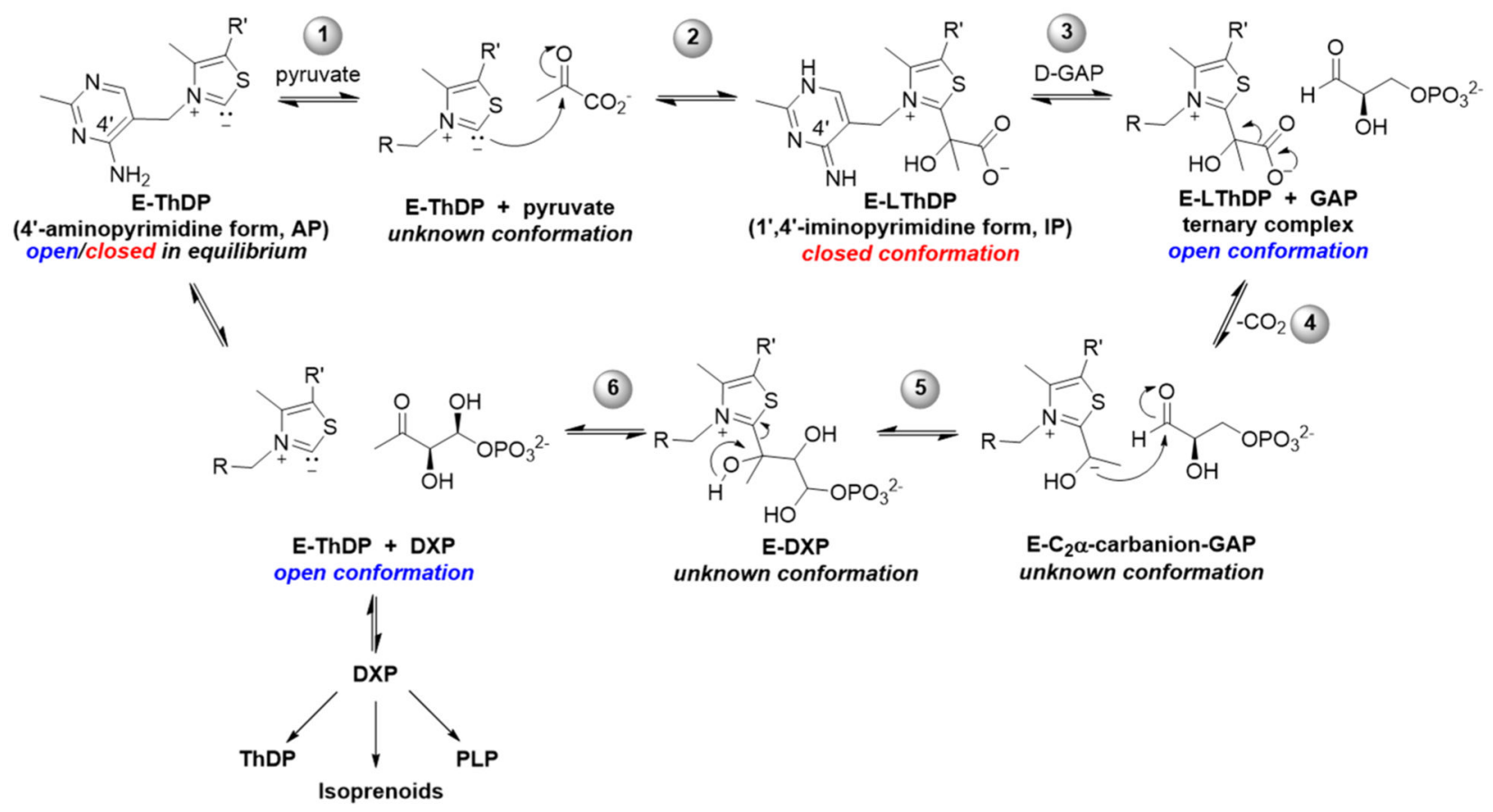

Figure 1.

DXP synthase catalyzes the ThDP-dependent formation of DXP, which is an intermediate in thee essential bacterial biosynthesis pathways. $\mathrm{E}=\mathrm{DXPS}$. $\mathrm{R}=4^{\prime}$-amino-2-methyl-5pyrimidyl. $\mathrm{R}^{\prime}=\beta$-hydroxyethyldiphosphate. 
A)

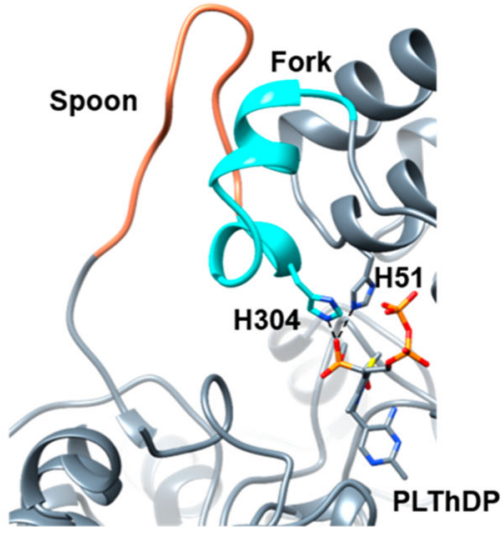

C)

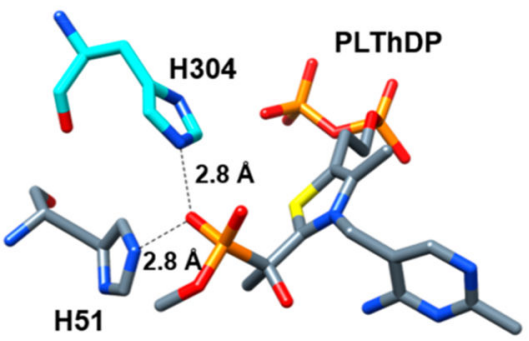

B)

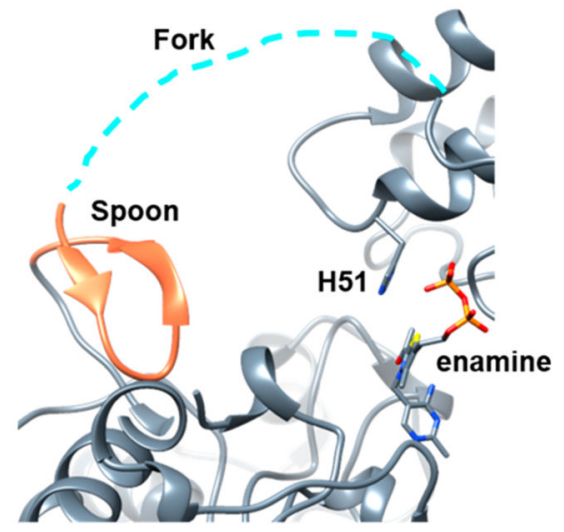

D)

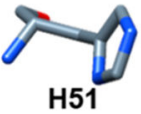

Figure 2.

D. radiodurans DXP synthase in the (A and C) predecarboxylation and (B and D) postdecarboxylation states illustrating the conformational change of the fork (teal, unresolved in the open structure) and spoon (orange) regions from the closed PLThDPbound structure to the open postdecarboxylation (shown as the enamine) structure. (C) Interactions of H51 and H304 (E. coli DXP synthase H49 and H299, respectively) with the phosphonyl moiety of PLThDP (and, by extension, the carboxyl moiety of LThDP) in the closed conformation. (D) Movement of the spoon and fork motifs away from the active site in the open conformation removes H304 from the active site. Prepared in UCSF Chimera. 
A)

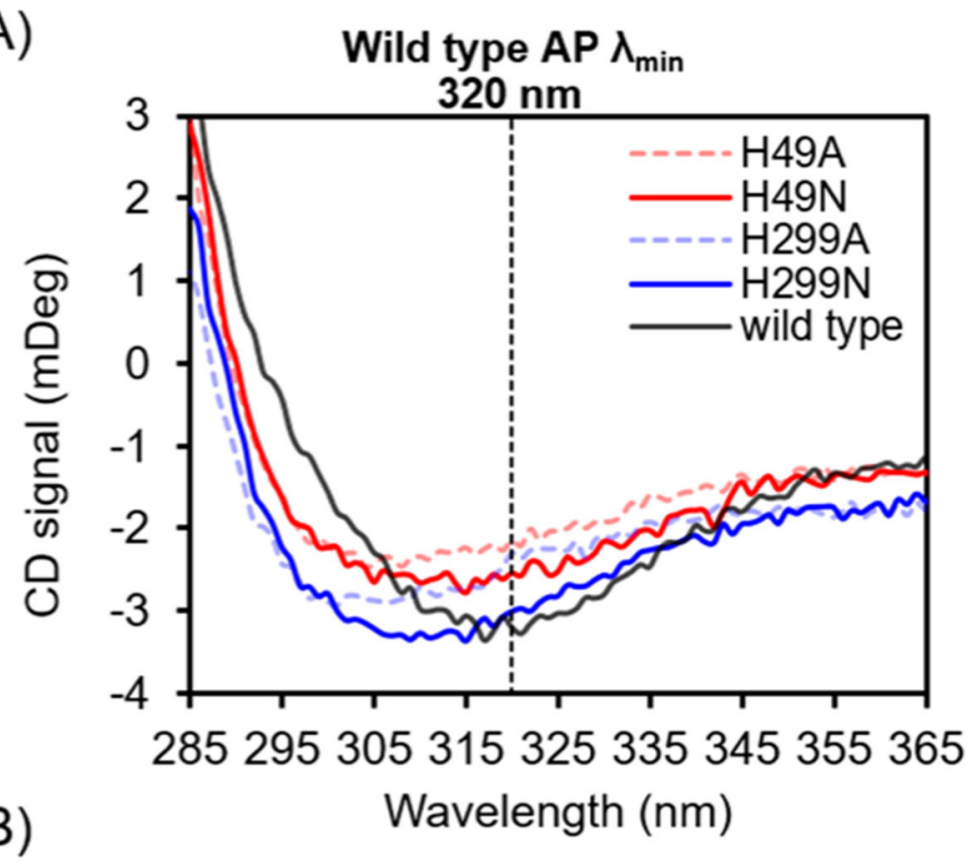

\begin{tabular}{|c|c|}
\hline Enzyme & AP $\boldsymbol{\Lambda}_{\text {min }}$ range $(\mathbf{n m})$ \\
\hline Wild Type & $317-322$ \\
\hline $\mathrm{H} 49 \mathrm{~A}$ & $308-310$ \\
\hline $\mathrm{H} 49 \mathrm{~N}$ & $311-315$ \\
\hline $\mathrm{H} 299 \mathrm{~A}$ & $301-303$ \\
\hline $\mathrm{H} 299 \mathrm{~N}$ & $301-309$ \\
\hline
\end{tabular}

Figure 3.

(A) Overlay of the CD spectra of wild type, H49A, H49N, H299A, and H299N DXP synthase in the resting, ThDP-bound state. (B) Comparison of the ranges of AP $\lambda_{\min }$ for wild type DXP synthase and its variants. 
A)

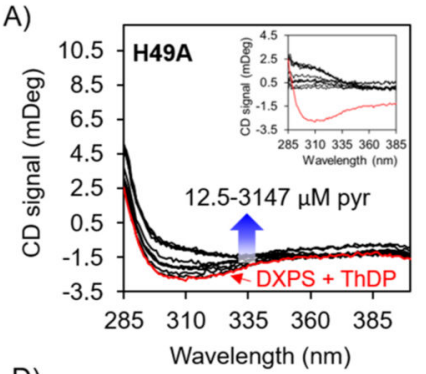

D)

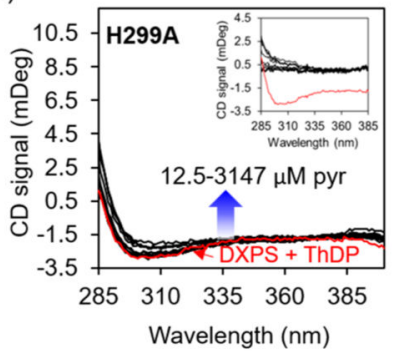

B)

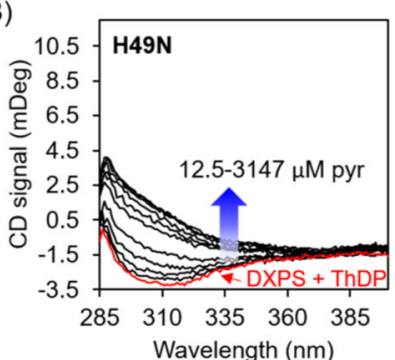

E)

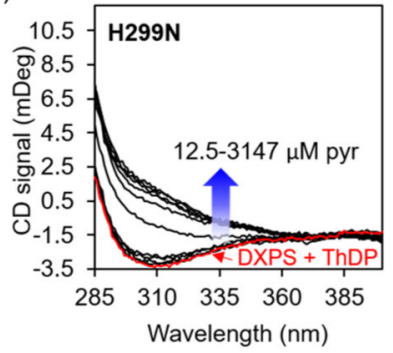

C)

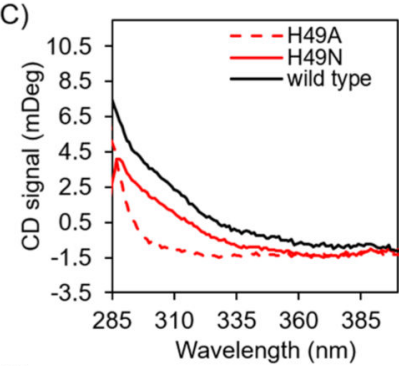

F)

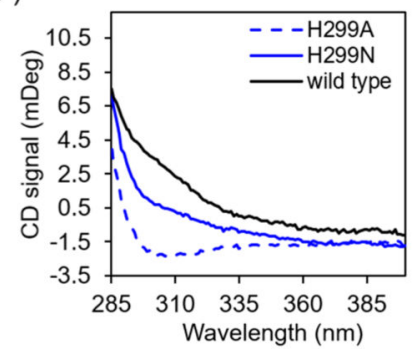

Figure 4.

Titration of pyruvate onto (A) H49A, (B) H49N, (D) H299A, and (E) H299N monitored by $\mathrm{CD}$ under anaerobic conditions. Insets illustrate enzyme-subtracted spectra for H49A and H299A. Overlay of CD spectra of (C) H49A variants and the wild type or (F) H299 variants and the wild type in the presence of a saturating pyruvate concentration indicating a lower signal intensity for LThDP accumulation on all variants compared to the wild type and on Ala variants compared to Asn variants at the same position. 
A)
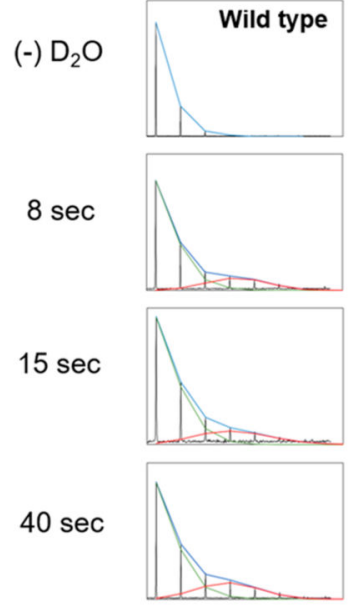

$1 \mathrm{~min}$

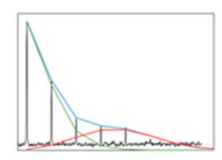

$3 \min$

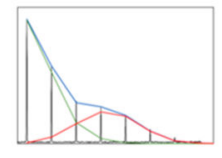

$5 \mathrm{~min}$

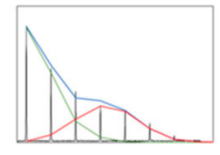

$10 \mathrm{~min}$

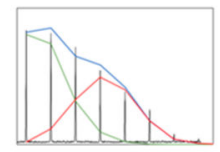

$30 \mathrm{~min}$

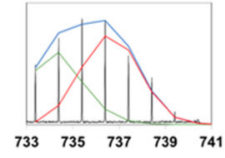

B)
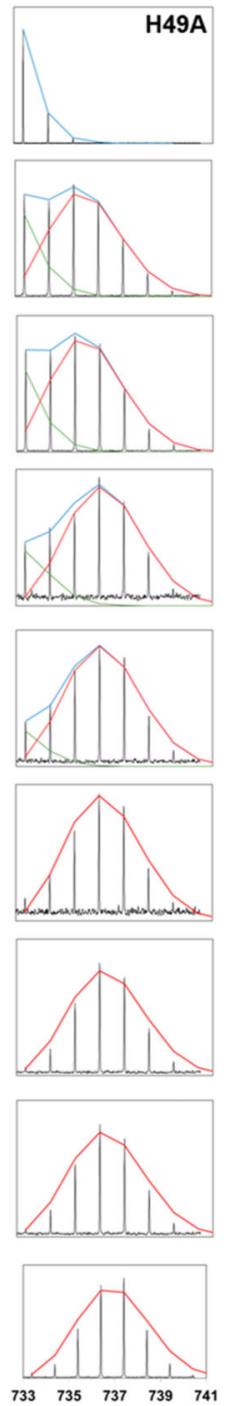

C)
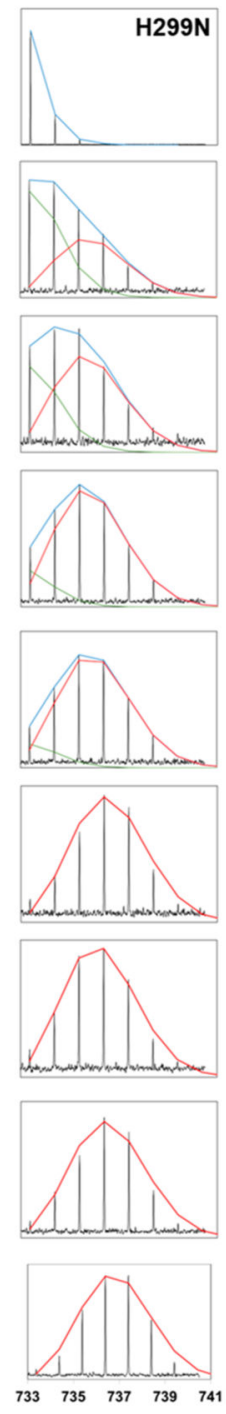

Figure 5.

Representative $m / z$ plots of HDX time courses of (A) wild type, (B) H49A, and (C) H299N in the absence of ligands on peptide 51-58. Deconvolution of the bimodal peak (blue) reveals a lower-mass envelope (green) that represents a closed state and a higher-mass envelope (red) that represents the open state. H49A and $\mathrm{H} 49 \mathrm{~N}$ are fully deuterated within 3 min, which is significantly faster than the wild type, which is still not fully exchanged by 30 $\min$. 
A)

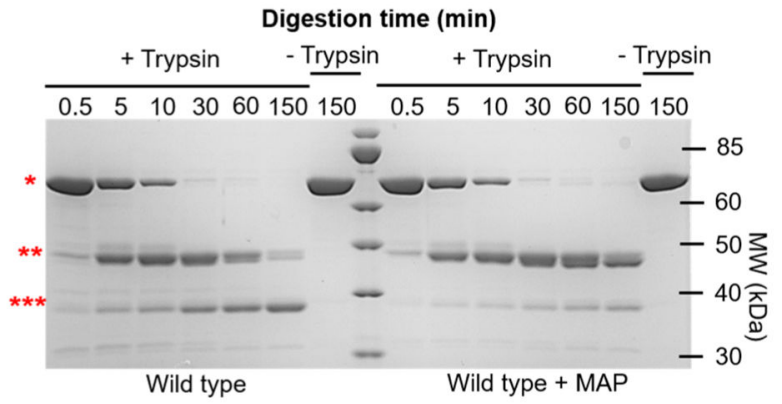

B)

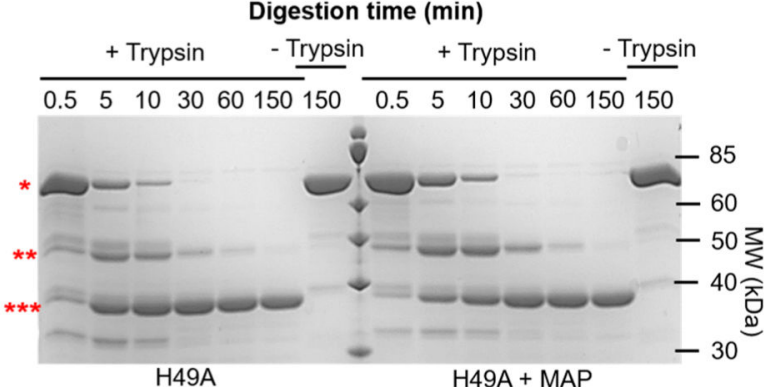

C)

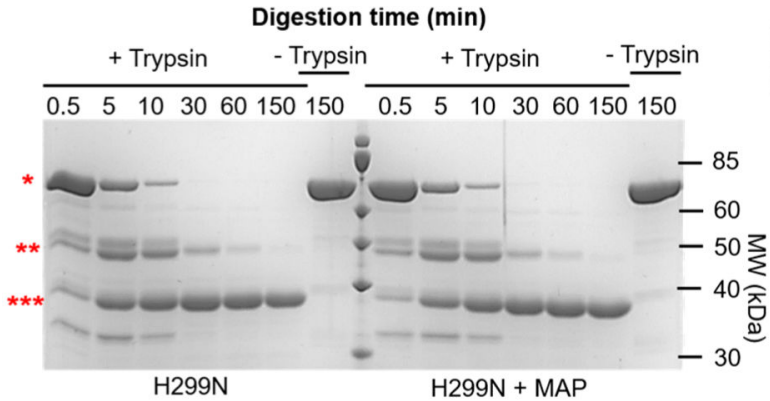

D)

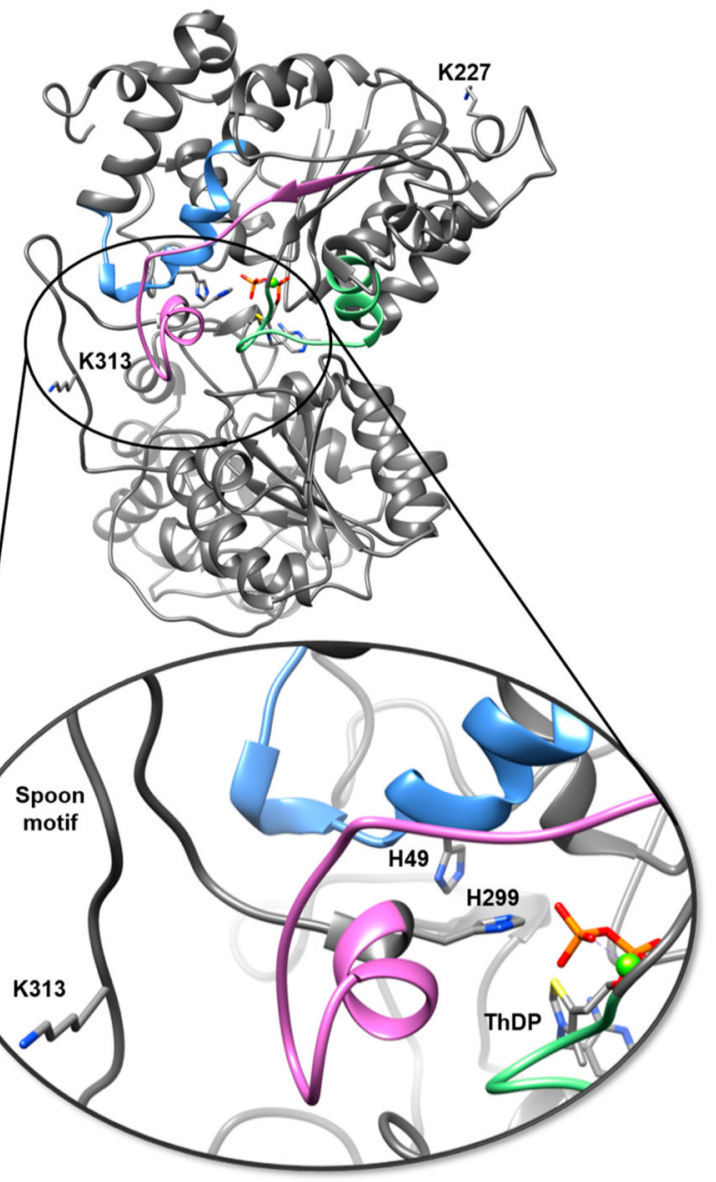

Figure 6.

Anaerobic limited trypsinolysis of (A) wild type, (B) H49A, and (C) H299N in the absence (left) and presence (right) of MAP over time. Full length DXP synthase (one asterisk), cleavage product 1 (44 kDa, two asterisks, cleavage at K227), and cleavage product 2 (34 $\mathrm{kDa}$, three asterisks, cleavage at K313) are indicated. (D) Closed model of E. coli DXP synthase. ${ }^{18}$ The positions of the two major trypsin cleavage sites as well at His49, His299, and ThDP are shown as sticks colored by element. The three flexible regions observed by HDX-MS are colored blue (residues 42-58), green (residues 182-199), and pink (residues 278-298). ${ }^{18}$ 
A) Digestion time (min)

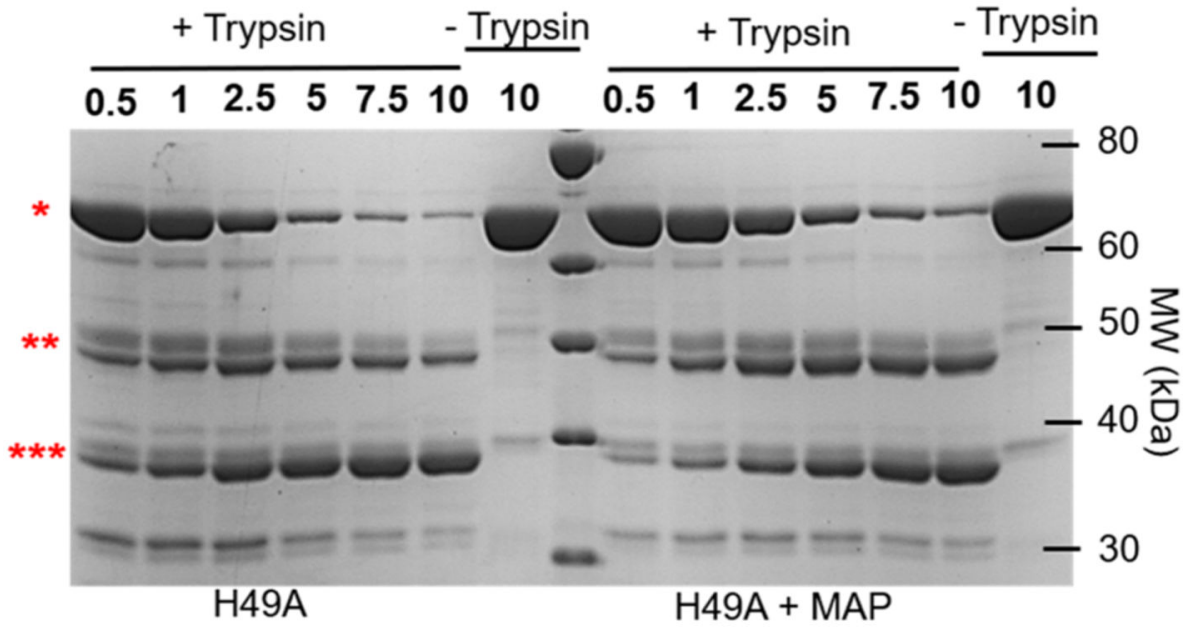

B) Digestion time (min)

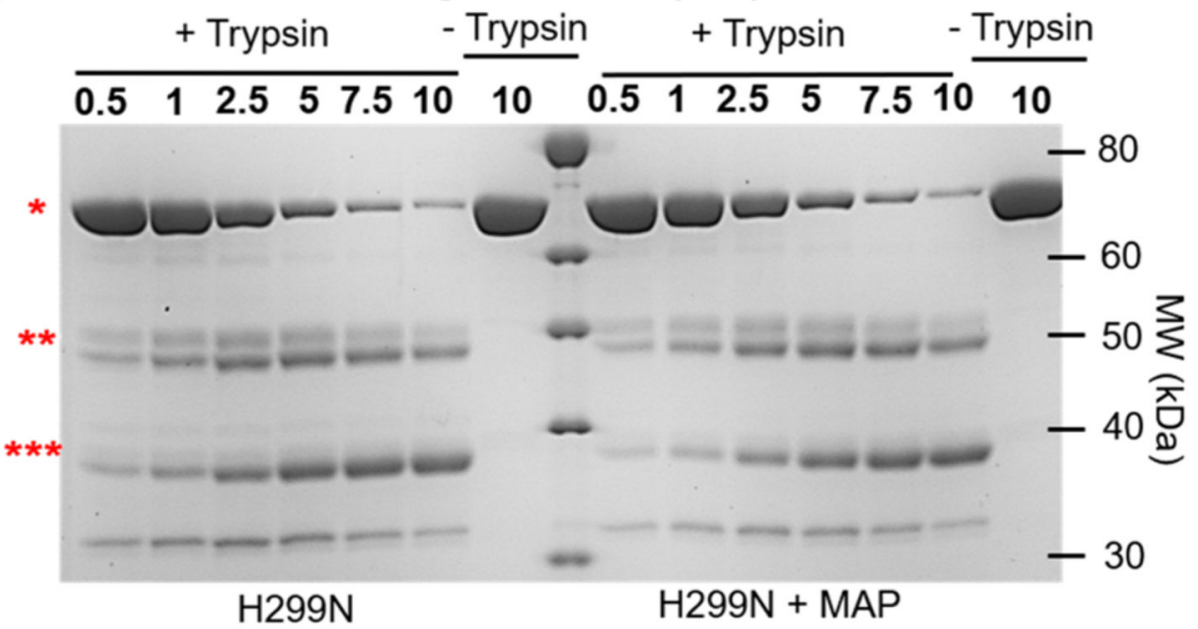

Figure 7.

Expanded limited trypsinolysis time course of (A) H49A and (B) H299N in the absence (left) and presence (right) of MAP. Full length DXP synthase (one asterisk), cleavage product 1 (44 kDa, two asterisks, cleavage at K227), and cleavage product 2 (34 kDa, three asterisks, cleavage at K313). Subtle accumulation over $10 \mathrm{~min}$ of the $44 \mathrm{kDa}$ peptide is apparent when H49A and H299N are preincubated with a saturating MAP concentration in contrast to limited trypsinolysis of the wild type under the same conditions in which accumulation of the $44 \mathrm{kDa}$ peptide is sustained over $150 \mathrm{~min}$ (Figure 6A). 


\section{롤 \\ 골}

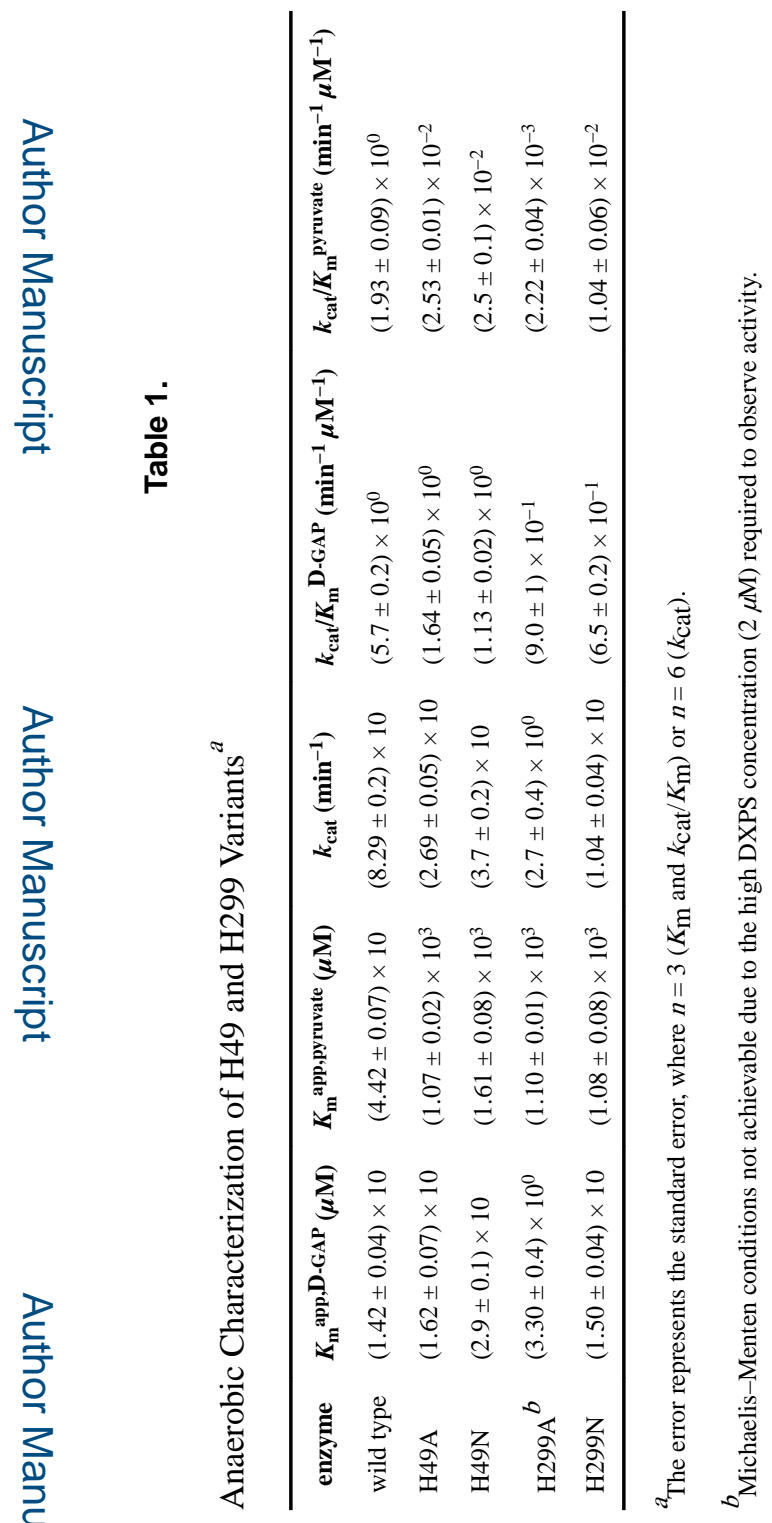

Biochemistry. Author manuscript; available in PMC 2019 December 11. 


\section{로을}

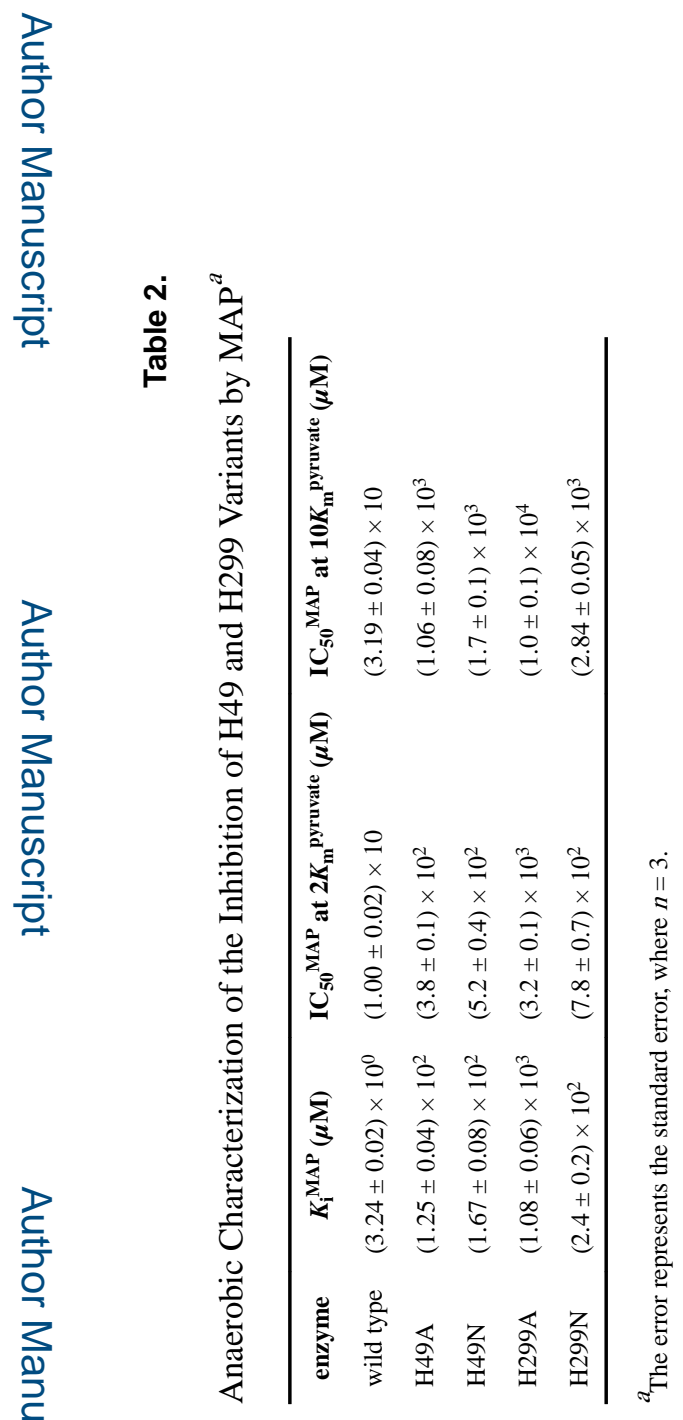




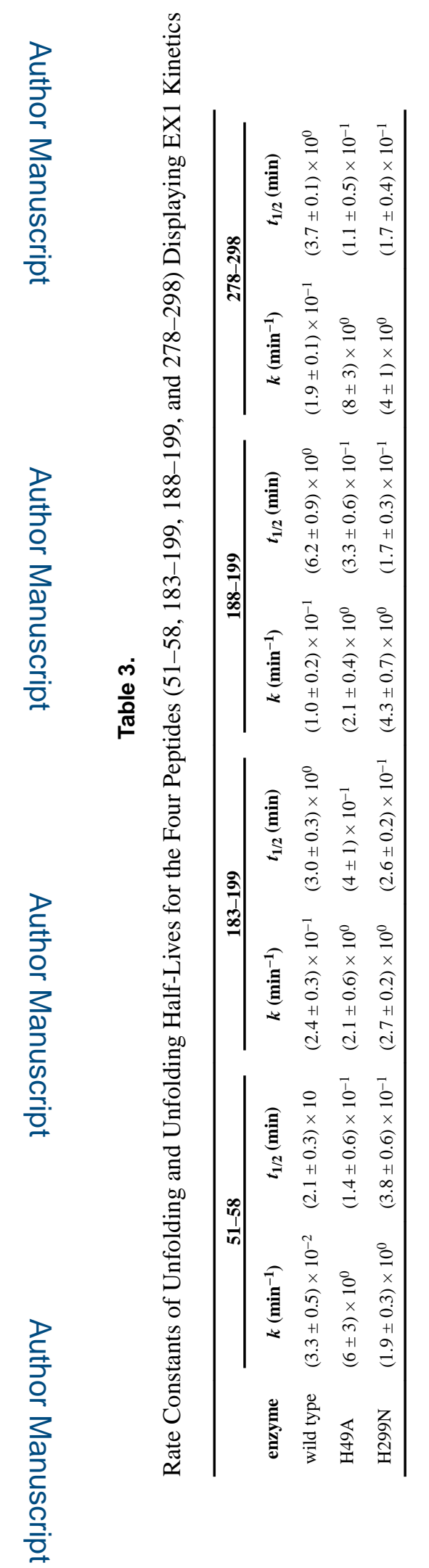

Biochemistry. Author manuscript; available in PMC 2019 December 11. 